\title{
An Improved Interpolating Element-Free Galerkin Method Based on Nonsingular Weight Functions
}

\author{
F. X. Sun, ${ }^{1,2}$ C. Liu, ${ }^{1}$ and Y. M. Cheng ${ }^{1}$ \\ ${ }^{1}$ Shanghai Institute of Applied Mathematics and Mechanics, Shanghai University, Shanghai 200072, China \\ ${ }^{2}$ Faculty of Science, Ningbo University of Technology, Ningbo 315016, China \\ Correspondence should be addressed to Y. M. Cheng; ymcheng@shu.edu.cn
}

Received 23 December 2013; Accepted 21 January 2014; Published 2 March 2014

Academic Editor: Miaojuan Peng

Copyright (c) 2014 F. X. Sun et al. This is an open access article distributed under the Creative Commons Attribution License, which permits unrestricted use, distribution, and reproduction in any medium, provided the original work is properly cited.

\begin{abstract}
Based on the moving least-squares (MLS) approximation, an improved interpolating moving least-squares (IIMLS) method based on nonsingular weight functions is presented in this paper. Then combining the IIMLS method and the Galerkin weak form, an improved interpolating element-free Galerkin (IIEFG) method is presented for two-dimensional potential problems. In the IIMLS method, the shape function of the IIMLS method satisfies the property of Kronecker $\delta$ function, and there is no difficulty caused by singularity of the weight function. Then in the IIEFG method presented in this paper, the essential boundary conditions are applied naturally and directly. Moreover, the number of unknown coefficients in the trial function of the IIMLS method is less than that of the MLS approximation; then under the same node distribution, the IIEFG method has higher computational precision than element-free Galerkin (EFG) method and interpolating element-free Galerkin (IEFG) method. Four selected numerical examples are presented to show the advantages of the IIMLS and IIEFG methods.
\end{abstract}

\section{Introduction}

In recent years, meshless (or meshfree) method has become very attractive to solve science and engineering problems without meshes. The most important common feature of meshless methods is that its approximation function is constructed entirely based on a set of scattered nodes without employing a mesh. Then some complicated problems, such as the large deformation and crack growth problems in mechanics, can be simulated with the method without the remeshing techniques [1-3].

Many meshless methods have been developed, such as element-free Galerkin (EFG) method [4-8], meshless local Petrov-Galerkin (MLPG) method [9], reproducing kernel particle method (RKPM) [10-13], complex variable meshless method [14-23], meshless manifold method [24-30], the mesh-free reproducing kernel particle Ritz method [31], finite point method (FPM) [32], radial basis functions (RBF) method [33, 34], boundary element-free method (BEFM) [35-40], boundary node method [41], and local boundary integral equation (LBIE) method $[42,43]$.
Moving least-square (MLS) approximation is an important method to form the shape functions in meshless methods, such as the EFG, LBIE, and MLPG methods. The MLS approximation was firstly introduced by Shepard [44] and then extended by Lancaster and Salkauskas for surface generation problems [45]. The shape function that is formed with MLS approximation can obtain a solution with high precision.

There exists a disadvantage in the MLS approximation in which its shape function does not satisfy the property of Kronecker $\delta$ function. Then the meshless methods based on the MLS approximation cannot apply the essential boundary conditions directly and easily. The essential boundary conditions need to be introduced by additional approaches, such as Lagrange multipliers [4] and penalty methods [46]. However, for Lagrange multipliers, the corresponding discrete system will introduce additional unknowns which are not directly associated with the solution themselves. And for penalty methods, the optimal value of penalty factor always affects the accuracy of the final solution. 
To overcome this disadvantage, Most and Bucher designed a regularized weight function with a regularization parameter $\varepsilon$, with which the MLS approximation can almost fulfill the interpolation and boundary conditions with high accuracy [47]. Thomas enhanced the regularized weight function to obtain a true interpolation of the MLS approximation [48]. Sergio obtained a special weight function using a normalization based on the Shepard interpolation to fulfill the interpolation [49].

Another possible approach for this disadvantage is the interpolating moving least-squares (IMLS) method presented by Lancaster and Salkauskas [45]. The IMLS method is established based on the MLS approximation by using singular weight functions. The shape function of the IMLS method satisfies the property of Kronecker $\delta$ function. Thus, the meshless methods based on the IMLS method can apply the essential boundary condition directly without any additional numerical effort. Based on the IMLS method, Kaljević and Saigal [50] presented an improved formulation of the element-free Galerkin (EFG) method, in which the boundary condition is applied directly. Ren simplified the expression of the shape function of the IMLS method and then presented the interpolating element-free Galerkin (IEFG) method and interpolating boundary element-free (IBEF) method for twodimensional potential and elasticity problems [51-54].

Certainly a disadvantage of the IMLS method is that its weight function is singular at nodes. It complicates the computation of the inverse of the singular matrix, and it causes many difficulties to obtain the derivatives of the approximation function in the IMLS method. To overcome the singularity, Netuzhylov presented the perturbation technique in the IMLS method by using a small positive number $\varepsilon$ within the weight function matrix [55]. However, the correct value of number $\varepsilon$ is also hard to be given, and it always affects the accuracy of the final solution. Based on the IMLS method, Cheng and so forth improved the interpolating moving leastsquares method with nonsingular weight function $[56,57]$. However, there exists a complicated function $v\left(\mathbf{x}, \mathbf{x}_{I}\right)$ in the improved method, and the computation of this function needs much CPU time. And then the computing speed of the improved method is lower than that of the IMLS method and MLS approximation.

In this paper, based on the MLS approximation and IMLS method, an improved interpolating moving leastsquares (IIMLS) method with nonsingular weight functions is presented. Compared with the IMLS method presented by Lancaster and Salkauskas, the weight function used in the IIMLS method is nonsingular at any points, and any weight function used in the MLS approximation can be chosen as the weight function of the IIMLS method. Then the IIMLS method can overcome the difficulties caused by singularity of the weight function as in the IMLS method. Compared with the shape function of MLS approximation, the shape function of the IIMLS method satisfies the property of Kronecker $\delta$ function. Then, the meshless method based on the IIMLS method can apply the essential boundary condition directly and easily without any additional approaches. And the number of unknown coefficients in the trial function of the IIMLS method is less than that in the trial function of the MLS approximation. Then fewer nodes are needed in the local influence domain in the IIMLS method than in the MLS approximation. Therefore, under the same node distribution, the IIMLS method has higher computational precision than the MLS approximation.

Based on the IIMLS method and the Galerkin weak form, an improved interpolating element-free Galerkin (IIEFG) method is presented for two-dimensional potential problems. Compared with the conventional EFG method, the essential boundary conditions in the IIEFG method are applied naturally and directly. As there are fewer coefficients in the trial function of the IIMLS method than that in the MLS approximation, fewer nodes are selected in the entire domain in the IIEFG method than in the conventional EFG method; then under the same node distribution, the IIEFG method has higher computational precision than elementfree Galerkin (EFG) method and interpolating element-free Galerkin (IEFG) method. Four selected numerical examples are presented to show the advantages of the IIMLS and IIEFG methods.

\section{The Improved Interpolating Moving Least-Squares Method}

The improved interpolating moving least-squares (IIMLS) method is presented in this section based on nonsingular weight functions.

Suppose $p_{1}(\mathbf{x}) \equiv 1, p_{2}(\mathbf{x}), \ldots, p_{m}(\mathbf{x})$ be given basis functions. In order to let the shape function of the IIMLS method, which can apply any nonsingular weight function used in the MLS approximation, satisfy the property of Kronecker $\delta$ function, we firstly generate a set of new local basis functions from these given basis functions.

Let $\rho_{I}$ denote the radius of the domain of influence of node and let $s_{I}=m_{I}(\mathbf{x})\left\|\mathbf{x}-\mathbf{x}_{I}\right\|$, where $m_{I}(\mathbf{x})=m\left(\mathbf{x}-\mathbf{x}_{I}\right) \epsilon$ $C^{l}(\Omega)$ satisfying $m_{I}(\mathbf{x})>0$ for $\left\|\mathbf{x}-\mathbf{x}_{I}\right\|<\rho_{I}$ and $m_{I}(\mathbf{x})=0$ for $\left\|\mathbf{x}-\mathbf{x}_{I}\right\| \geq \rho_{I}$. For a given point $\mathbf{x} \in \Omega$, let

$$
\widetilde{p}_{i}(\overline{\mathbf{x}})_{\mathbf{x}}=p_{i}(\overline{\mathbf{x}})-\sum_{I=1}^{n} v\left(\mathbf{x}, \mathbf{x}_{I}\right) p_{i}\left(\mathbf{x}_{I}\right), \quad i=1,2, \ldots, m,
$$

where $\overline{\mathbf{x}}$ is the point in the local approximation of $\mathbf{x}, \mathbf{x}_{I}(I=$ $1,2, \ldots, n)$ are the nodes with domains of influence that cover the point $\mathbf{x}$, and

$$
v\left(\mathbf{x}, \mathbf{x}_{I}\right)=\frac{s_{L}^{\alpha} s_{I}^{-\alpha}}{1+s_{L}^{\alpha} \sum_{J=1, J \neq L}^{n} s_{J}^{-\alpha}}
$$

where the parameter $\alpha$ is an even positive integer and $s_{L}$ denotes the distance from $\mathbf{x}$ to the closest support node whose domain of influence covers $\mathbf{x}$.

The function $v\left(\mathbf{x}, \mathbf{x}_{I}\right)$ in this paper is easier than the corresponding function in $[56,57]$. Then the improved method in this paper has high computing speed. The function $v\left(\mathbf{x}, \mathbf{x}_{I}\right) \in C^{l}(\Omega)$ and satisfies

(a) $v\left(\mathbf{x}_{I}, \mathbf{x}_{J}\right)=\delta_{I J}$;

(b) $\sum_{I=1}^{n} v\left(\mathbf{x}, \mathbf{x}_{I}\right)=1, \forall \mathbf{x} \in \Omega$; 
(c) $0 \leq v\left(\mathbf{x}, \mathbf{x}_{I}\right) \leq 1, \forall \mathbf{x} \in \Omega$, and $v\left(\mathbf{x}, \mathbf{x}_{I}\right)=0$ if and only if $\mathbf{x}=\mathbf{x}_{J}$ and $J \neq I$

(d) $\partial v\left(\mathbf{x}_{I}, \mathbf{x}_{J}\right) / \partial \mathbf{x}=0$.

And then a local function is proposed by performing the same transformation to $u(\mathbf{x})$; that is,

$$
\widetilde{u}(\overline{\mathbf{x}})_{\mathbf{x}}=u(\overline{\mathbf{x}})-\sum_{I=1}^{n} v\left(\mathbf{x}, \mathbf{x}_{I}\right) u\left(\mathbf{x}_{I}\right) .
$$

Next the approximation function of the new local function $\tilde{u}(\overline{\mathbf{x}})_{x}$ is obtained with the MLS approximation with the new local basis functions.

A local approximation function of $\widetilde{u}(\overline{\mathbf{x}})_{x}$ is defined as

$$
\widetilde{u}^{h}(\overline{\mathbf{x}})_{\mathbf{x}}=\sum_{i=1}^{m} \widetilde{p}_{i}(\overline{\mathbf{x}})_{\mathbf{x}} \widetilde{a}_{i}(\mathbf{x})
$$

where $\widetilde{a}_{i}(\mathbf{x})$ are the unknown coefficients of the new basis.

From (2), we have

$$
\sum_{I=1}^{n} v\left(\mathbf{x}, \mathbf{x}_{I}\right)=\frac{\sum_{I=1}^{n} s_{L}^{\alpha} s_{I}^{-\alpha}}{1+s_{L}^{\alpha} \sum_{J=1, J \neq L}^{n} s_{J}^{-\alpha}}=1
$$

Then there exists

$$
\widetilde{p}_{1}(\overline{\mathbf{x}})_{\mathbf{x}} \equiv 1-\sum_{I=1}^{n} v\left(\mathbf{x}, \mathbf{x}_{I}\right)=0
$$

Then

$$
\widetilde{u}^{h}(\overline{\mathbf{x}})_{\mathbf{x}}=\sum_{i=2}^{m} \widetilde{p}_{i}(\overline{\mathbf{x}})_{\mathbf{x}} \widetilde{a}_{i}(\mathbf{x})
$$

The $m-1$ unknown coefficients $\tilde{a}_{i}(\mathbf{x}), i=2,3, \ldots, m$, can be obtained by using the weighted least-square method. By the similar derivation as in [56], we have

$$
\widetilde{\mathbf{a}}(\mathbf{x})=\widetilde{\mathbf{A}}^{-1}(\mathbf{x}) \widetilde{\mathbf{B}}(\mathbf{x}) \mathbf{u},
$$

where

$$
\begin{gathered}
\tilde{\mathbf{a}}^{T}(\mathbf{x})=\left(\widetilde{a}_{2}(\mathbf{x}), \widetilde{a}_{3}(\mathbf{x}), \ldots, \tilde{a}_{m}(\mathbf{x})\right) \\
\mathbf{u}^{T}=\left(u\left(\mathbf{x}_{1}\right), u\left(\mathbf{x}_{2}\right), \ldots, u\left(\mathbf{x}_{n}\right)\right) \\
\widetilde{\mathbf{A}}(\mathbf{x})=\widetilde{\mathbf{P}}^{T}(\mathbf{x}) \mathbf{W}(\mathbf{x}) \widetilde{\mathbf{P}}(\mathbf{x}), \\
\widetilde{\mathbf{B}}(\mathbf{x})=\widetilde{\mathbf{P}}^{T}(\mathbf{x}) \mathbf{W}(\mathbf{x})(\mathbf{E}-\mathbf{V}(\mathbf{x})), \\
\mathbf{W}(\mathbf{x})=\left[\begin{array}{cccc}
w\left(\mathbf{x}-\mathbf{x}_{1}\right) & 0 & \cdots & 0 \\
0 & w\left(\mathbf{x}-\mathbf{x}_{2}\right) & \cdots & 0 \\
\vdots & \vdots & \ddots & \vdots \\
0 & 0 & \cdots & w\left(\mathbf{x}-\mathbf{x}_{n}\right)
\end{array}\right],
\end{gathered}
$$

$$
\begin{gathered}
\widetilde{\mathbf{P}}(\mathbf{x})=\left[\begin{array}{cccc}
\tilde{p}_{2}\left(\mathbf{x}_{1}\right)_{\mathbf{x}} & \tilde{p}_{3}\left(\mathbf{x}_{1}\right)_{\mathbf{x}} & \cdots & \tilde{p}_{m}\left(\mathbf{x}_{1}\right)_{\mathbf{x}} \\
\tilde{p}_{2}\left(\mathbf{x}_{2}\right)_{\mathbf{x}} & \widetilde{p}_{3}\left(\mathbf{x}_{2}\right)_{\mathbf{x}} & \cdots & \tilde{p}_{m}\left(\mathbf{x}_{2}\right)_{\mathbf{x}} \\
\vdots & \vdots & \ddots & \vdots \\
\tilde{p}_{2}\left(\mathbf{x}_{n}\right)_{\mathbf{x}} & \tilde{p}_{3}\left(\mathbf{x}_{n}\right)_{\mathbf{x}} & \cdots & \tilde{p}_{m}\left(\mathbf{x}_{n}\right)_{\mathbf{x}}
\end{array}\right], \\
\mathbf{V}(\mathbf{x})=\left[\begin{array}{cccc}
v\left(\mathbf{x}, \mathbf{x}_{1}\right) & v\left(\mathbf{x}, \mathbf{x}_{2}\right) & \cdots & v\left(\mathbf{x}, \mathbf{x}_{n}\right) \\
v\left(\mathbf{x}, \mathbf{x}_{1}\right) & v\left(\mathbf{x}, \mathbf{x}_{2}\right) & \cdots & v\left(\mathbf{x}, \mathbf{x}_{n}\right) \\
\vdots & \vdots & \ddots & \vdots \\
v\left(\mathbf{x}, \mathbf{x}_{1}\right) & v\left(\mathbf{x}, \mathbf{x}_{2}\right) & \cdots & v\left(\mathbf{x}, \mathbf{x}_{n}\right)
\end{array}\right]_{n \times n}
\end{gathered}
$$

and $\mathbf{E}$ is an $n \times n$ identity matrix.

Then the approximation function of $u(\mathbf{x})$ can be obtained as

$$
u^{h}(\mathbf{x})=\Phi^{T}(\mathbf{x}) \mathbf{u}=\sum_{I=1}^{n} \Phi_{I}(\mathbf{x}) u\left(\mathbf{x}_{I}\right),
$$

where $\boldsymbol{\Phi}(\mathbf{x})$ is a matrix of shape function

$$
\begin{gathered}
\Phi^{T}(\mathbf{x})=\left(\Phi_{1}(\mathbf{x}), \Phi_{2}(\mathbf{x}), \ldots, \Phi_{n}(\mathbf{x})\right) \\
=\mathbf{v}^{T}(\mathbf{x})+\mathbf{g}^{T}(\mathbf{x}) \widetilde{\mathbf{A}}^{-1}(\mathbf{x}) \widetilde{\mathbf{B}}(\mathbf{x}), \\
\mathbf{v}^{T}(\mathbf{x})=\left(v\left(\mathbf{x}, \mathbf{x}_{1}\right), v\left(\mathbf{x}, \mathbf{x}_{2}\right), \ldots, v\left(\mathbf{x}, \mathbf{x}_{n}\right)\right), \\
\mathbf{g}^{T}(\mathbf{x})=\left(g_{2}(\mathbf{x}), g_{3}(\mathbf{x}), \ldots, g_{m}(\mathbf{x})\right), \\
g_{i}(\mathbf{x})=p_{i}(\mathbf{x})-\sum_{I=1}^{n} v\left(\mathbf{x}, \mathbf{x}_{I}\right) p_{i}\left(\mathbf{x}_{I}\right) .
\end{gathered}
$$

Equation (11) is the shape function of the IIMLS method, and then the IIMLS method is presented.

From properties of the function $v\left(\mathbf{x}, \mathbf{x}_{I}\right)$, the shape function of the IIMLS method certainly satisfies the property of Kronecker $\delta$ function; that is,

$$
\Phi_{I}\left(\mathbf{x}_{J}\right)=\delta_{I J}
$$

The linear combination of the basis functions can be exactly reproduced with the IIMLS method; that is, if we let

$$
u(\mathbf{x})=\beta_{1} p_{1}(\mathbf{x})+\beta_{2} p_{2}(\mathbf{x})+\cdots+\beta_{m} p_{m}(\mathbf{x}),
$$

where $\beta_{1}, \beta_{2}, \ldots, \beta_{m}$ are arbitrary constants, there exists

$$
u^{h}(\mathbf{x})=u(\mathbf{x}) .
$$

Compared with the MLS approximation, the shape function of the IIMLS method can satisfy the property of Kronecker $\delta$ function, and then the meshless method based on the IIMLS method can apply the essential boundary conditions directly. From (7), it can also be seen that the number of the unknown coefficients in the trial function of the IIMLS method is less than that in the trial function of the MLS approximation. Therefore, we can select fewer nodes in the meshless method based on the IIMLS method than that based on the MLS approximation. Hence, under 
the same node distribution, the IIMLS method has higher computational precision than the MLS approximation.

Compared with the IMLS method presented by Lancaster and Salkauskas, the nonsingular weight function is used in the IIMLS method. Then any weight function used in the MLS approximation can also be used in the IIMLS method. Then the IIMLS method can overcome the difficulties caused by the singularity of the weight function as in the IMLS method.

\section{The Improved Interpolating Element-Free Galerkin Method}

In this section, combining the IIMLS method and Galerkin weak form of the potential problems, the improved interpolating element-free Galerkin (IIEFG) method for the twodimensional potential problems is presented. Since the shape function of the IIMLS method satisfies the property of Kronecker $\delta$ function, the IIEFG method can apply the essential boundary conditions directly and easily.

Consider the following two-dimensional Poisson's equation:

$$
\nabla^{2} u(\mathbf{x})+b(\mathbf{x})=0, \quad \mathbf{x}=\left(x_{1}, x_{2}\right) \in \Omega,
$$

with boundary conditions of the Dirichlet type, that is,

$$
u(\mathbf{x})=\bar{u}(\mathbf{x}), \quad \mathbf{x} \in \Gamma_{u},
$$

or the Neumann type, that is,

$$
q(\mathbf{x})=\frac{\partial u(\mathbf{x})}{\partial \mathbf{n}}=\bar{q}(\mathbf{x}), \quad \mathbf{x} \in \Gamma_{q}
$$

where $u(\mathbf{x})$ is an unknown function, $b(\mathbf{x})$ is a known function, $\mathbf{n}$ is the unit outward normal to the boundary $\Gamma$, and $\bar{u}$ and $\bar{q}$ are, respectively, the prescribed values of the function $u(\mathbf{x})$ and its normal derivative over the boundary $\Gamma$. Notice that $\Gamma=\Gamma_{u} \cup \Gamma_{q}$.

The Galerkin weak form of (18)-(20) is

$$
\begin{gathered}
\int_{\Gamma_{q}} \delta u(\mathbf{x}) \bar{q}(\mathbf{x}) d \Gamma-\int_{\Omega} \delta(\nabla u)^{T} \nabla u d \Omega \\
+\int_{\Omega} \delta u(\mathbf{x}) b(\mathbf{x}) d \Omega=0
\end{gathered}
$$

where

$$
\nabla u=\left(\frac{\partial u}{\partial x_{1}}, \frac{\partial u}{\partial x_{2}}\right)^{T}=\left(u_{, 1}, u_{, 2}\right)^{T} .
$$

We employ $n_{t}$ nodes in the domain $\Omega$, and the union of their compact support domains $\Omega_{I}, I=1,2, \ldots, n_{t}$, must cover the whole domain $\Omega$.

From the IIMLS method, the unknown potential $u(\mathbf{x})$ at arbitrary field point $\mathbf{x}$ in the domain $\Omega$ can be expressed as

$$
u(\mathbf{x})=\Phi^{T}(\mathbf{x}) \mathbf{u}=\sum_{I=1}^{n} \Phi_{I}(\mathbf{x}) u\left(\mathbf{x}_{I}\right),
$$

where $n$ is the number of nodes whose compact support domains cover the point $\mathbf{x}$.

Then we have

$$
\nabla u=\nabla \Phi^{T}(\mathbf{x}) \mathbf{u}=\sum_{I=1}^{n} \nabla \Phi_{I}(\mathbf{x}) u\left(\mathbf{x}_{I}\right) \equiv \mathbf{B}^{T} \mathbf{u},
$$

where

$$
\mathbf{B}^{T}=\left[\begin{array}{llll}
\Phi_{1,1}(\mathbf{x}) & \Phi_{2,1}(\mathbf{x}) & \cdots & \Phi_{n, 1}(\mathbf{x}) \\
\Phi_{1,2}(\mathbf{x}) & \Phi_{2,2}(\mathbf{x}) & \cdots & \Phi_{n, 2}(\mathbf{x})
\end{array}\right] .
$$

Substituting (23) and (33) into (21) yields

$$
\begin{gathered}
\int_{\Gamma_{q}} \delta\left(\boldsymbol{\Phi}^{T}(\mathbf{x}) \mathbf{u}\right) \bar{q}(\mathbf{x}) d \Gamma-\int_{\Omega} \delta\left(\mathbf{B}^{T} \mathbf{u}\right)^{T}\left(\mathbf{B}^{T} \mathbf{u}\right) d \Omega \\
+\int_{\Omega} \delta\left(\boldsymbol{\Phi}^{T}(\mathbf{x}) \mathbf{u}\right) b(\mathbf{x}) d \Omega=0
\end{gathered}
$$

Then it follows from (26) that

$$
\begin{gathered}
\int_{\Gamma_{q}} \delta \mathbf{u}^{T} \cdot \Phi(\mathbf{x}) \bar{q}(\mathbf{x}) d \Gamma-\int_{\Omega} \delta \mathbf{u}^{T} \mathbf{B} \mathbf{B}^{T} \mathbf{u} d \Omega \\
+\int_{\Omega} \delta \mathbf{u}^{T} \boldsymbol{\Phi}(\mathbf{x}) b(\mathbf{x}) d \Omega=0 ;
\end{gathered}
$$

that is,

$$
\begin{gathered}
\delta \mathbf{u}^{T}\left[\int_{\Gamma_{q}} \boldsymbol{\Phi}(\mathbf{x}) \bar{q}(\mathbf{x}) d \Gamma-\left(\int_{\Omega} \mathbf{B B}^{T} d \Omega\right) \mathbf{u}\right. \\
\left.\quad+\int_{\Omega} \boldsymbol{\Phi}(\mathbf{x}) b(\mathbf{x}) d \Omega\right]=0 .
\end{gathered}
$$

Because the nodal test function $\delta \mathbf{u}$ is arbitrary, the final discretized equation is obtained as

$$
\begin{gathered}
\int_{\Gamma_{q}} \boldsymbol{\Phi}(\mathbf{x}) \bar{q}(\mathbf{x}) d \Gamma-\left(\int_{\Omega} \mathbf{B B}^{T} d \Omega\right) \mathbf{u} \\
+\int_{\Omega} \Phi(\mathbf{x}) b(\mathbf{x}) d \Omega=0 ;
\end{gathered}
$$

that is,

$$
\mathbf{K u}=\mathbf{F},
$$

where

$$
\begin{gathered}
\mathbf{K}_{I J}=\int_{\Omega} \nabla \Phi_{I}^{T} \cdot \nabla \Phi_{J} d \Omega \\
\mathbf{F}_{I}=\int_{\Gamma_{q}} \Phi_{I} \bar{q}(\mathbf{x}) d \Gamma+\int_{\Omega} \Phi_{I} b(\mathbf{x}) d \Omega .
\end{gathered}
$$

The shape function of the IIMLS method satisfies the property of Kronecker $\delta$ function, and then the essential boundary conditions can be applied directly. Substituting the boundary conditions of (19) into (30) directly, we can obtain the unknowns at nodes by solving (30). 


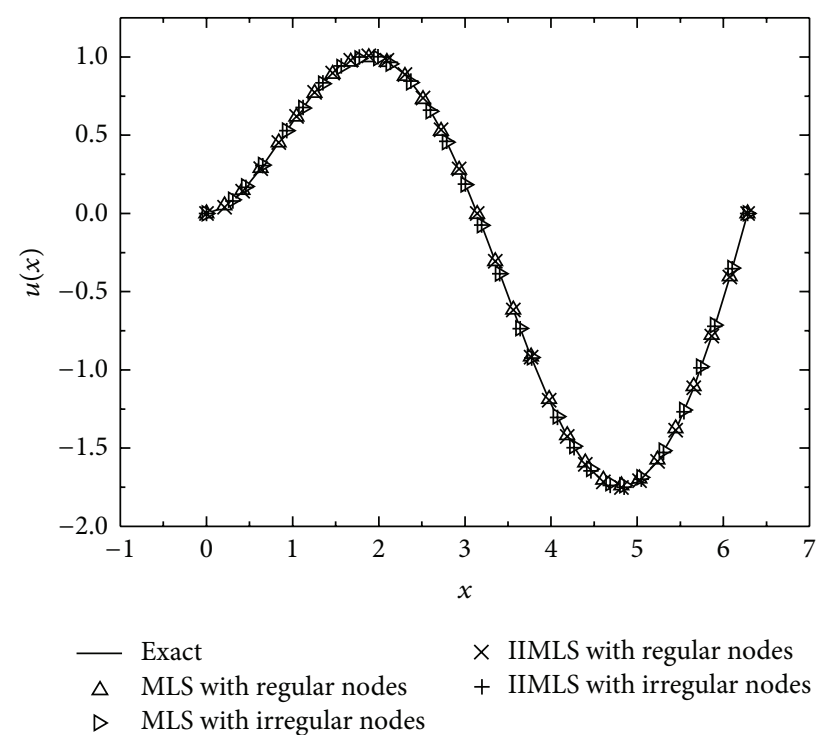

FIGURE 1: $u(x)$ obtained with the MLS and IIMLS methods under the regular and irregular node distributions.

Compared with the conventional EFG method based on the MLS approximation, the IIEFG method based on the IIMLS method can apply the essential boundary conditions directly and easily. And the number of the unknown coefficients in the trial function of the IIMLS method is less than that in the trial function of the MLS approximation. Hence, under the same node distribution, the IIEFG method has higher computational precision.

Compared with the IEFG method based on the IMLS method presented by Lancaster and Salkauskas, the IIEFG method applies the nonsingular weight function. Then the IIEFG method can overcome the difficulties caused by the singularity of the weight function as in the IEFG method. Then the IIEFG method also has higher computational precision.

\section{Numerical Examples}

The weight function plays an important role in the IIMLS method. Any weight function used in the MLS approximation can also be used in the IIMLS method. Then the cubic spline weight function, that is,

$$
w\left(\mathbf{x}-\mathbf{x}_{I}\right) \equiv w(r)= \begin{cases}\frac{2}{3}-4 r^{2}+4 r^{3}, & r \leq \frac{1}{2}, \\ \frac{4}{3}-4 r+4 r^{2}-\frac{4 r^{3}}{3}, & \frac{1}{2}<r \leq 1, \\ 0, & r>1,\end{cases}
$$

is used in the present analysis. Here

$$
r=\frac{d_{I}}{\rho_{I}}, \quad d_{I}=\left\|\mathbf{x}-\mathbf{x}_{I}\right\| .
$$

And the $m_{I}(\mathbf{x})$ is also chosen to be the cubic spline weight function.

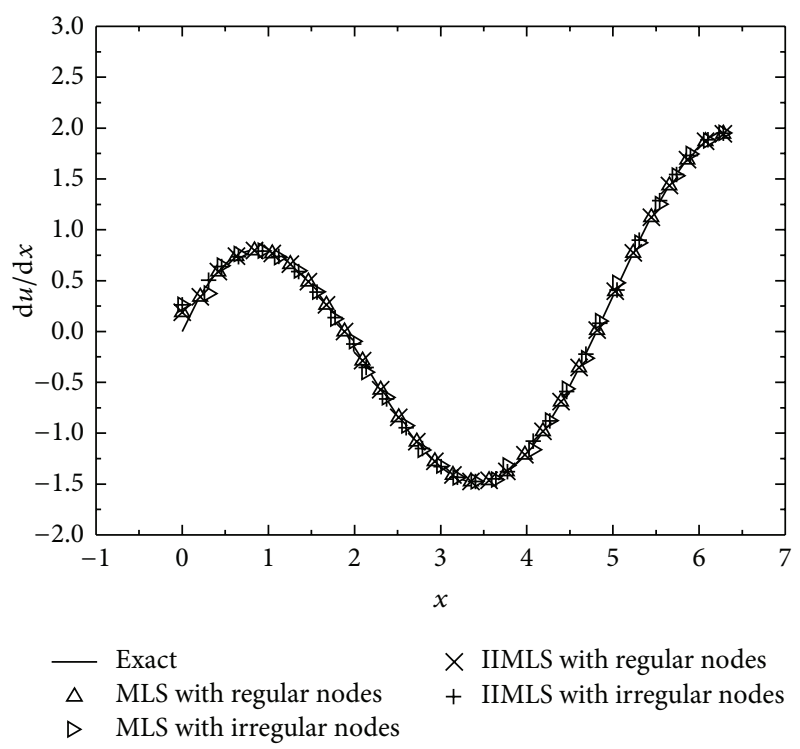

FIGURE 2: The derivatives of $u(x)$ obtained with the MLS and IIMLS methods under the regular and irregular node distributions.

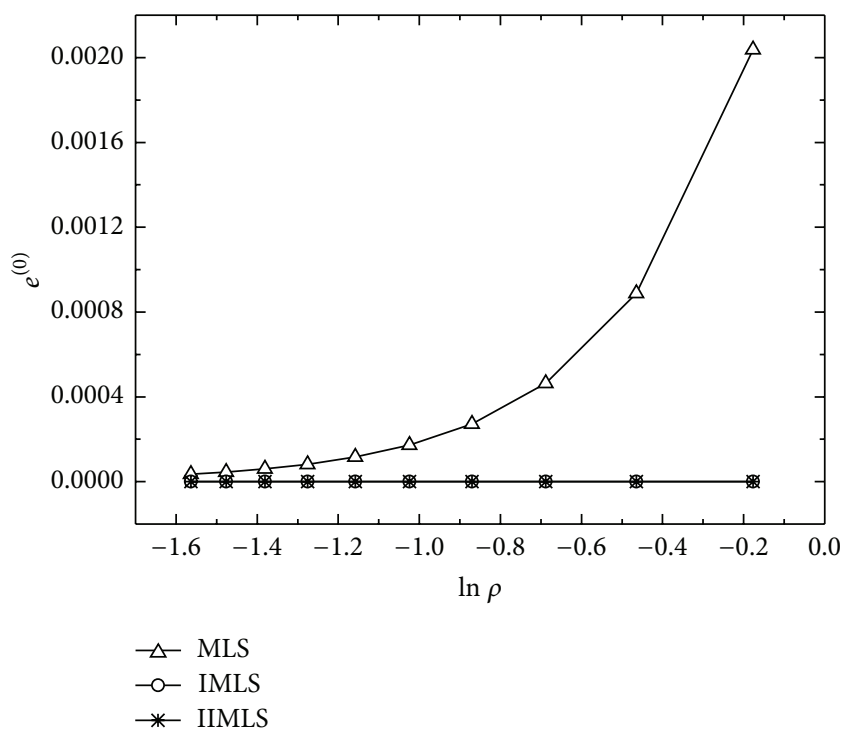

FIGURE 3: The error norms $e^{(0)}$ of $u(x)$ obtained with the MLS, IMLS, and IIMLS methods under the regular node distribution.

4.1. Examples of the IIMLS Method. In this section, two numerical examples are presented to show the advantages of the IIMLS method of this paper. In our examples, the interpolating approximation function $u^{h}(x)$ is constructed from a given function $u(x)$ with the IIMLS method. Let $\rho=$ $\max _{1 \leq I \leq n_{t}} \rho_{I}$, and the linear basis function is used in these examples of this section. Define the error norm

$$
e^{(\xi)}=\frac{1}{K} \sqrt{\frac{\sum_{I=1}^{K}\left[\partial^{(\xi)}\left[u_{I}^{h}-u_{I}\right]\right]^{2}}{\sum_{I=1}^{K}\left[\partial^{(\xi)} u_{I}\right]^{2}}},
$$




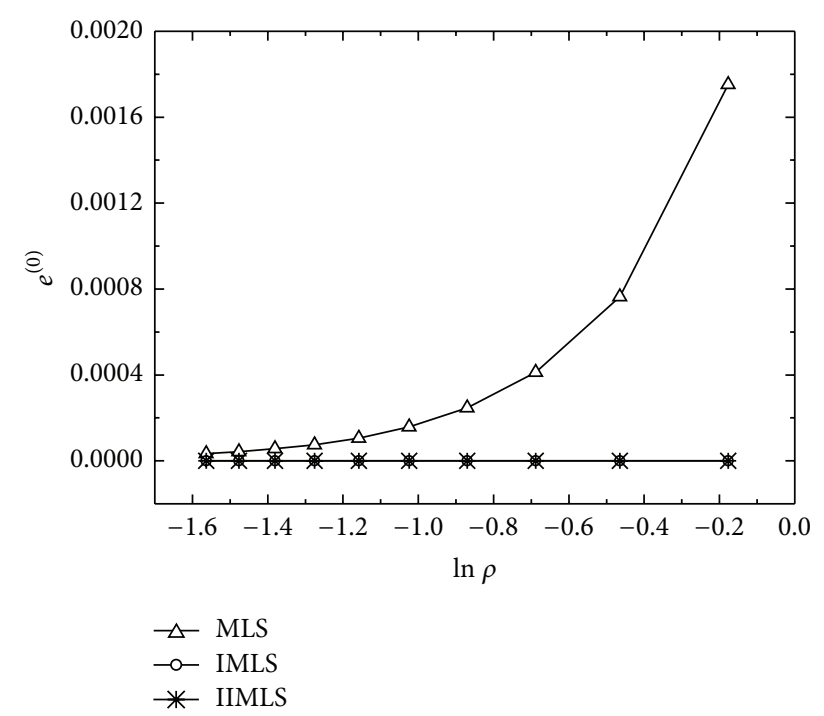

FIGURE 4: The error norms $e^{(0)}$ of $u(x)$ obtained with the MLS, IMLS, and IIMLS methods under the irregular node distribution.

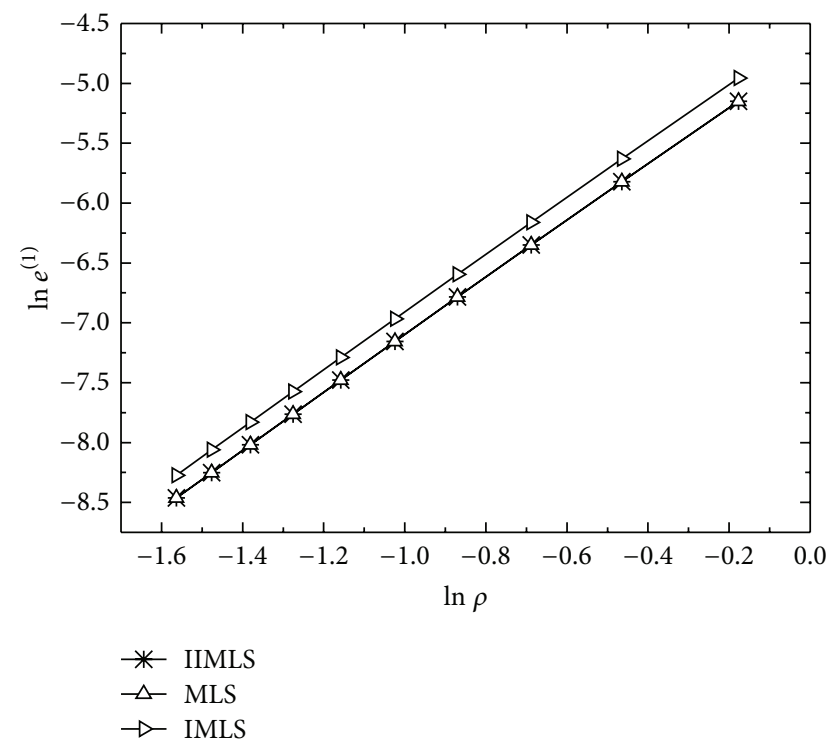

FIGURE 5: The error norms $e^{(1)}$ of the first derivative of $u(x)$ obtained with the MLS, IMLS, and IIMLS methods under the regular node distribution.

where $K$ is the number of nodes investigated, $\xi$ is a scalar constant and $\partial^{(\xi)} u=\left(d^{\xi} / d x^{\xi}\right) u$ in the one-dimensional space, and $\xi=(i, j)$ is a multi-index and $\partial^{(\xi)} u=\left(\partial^{i+j} / \partial x_{1}^{i} \partial x_{2}^{j}\right) u$ in the two-dimensional space.

The first example is considered in the one-dimensional space, and the given function is chosen to be $u(x)=$ $\sin x \ln (x+1), \Omega=[0,2 \pi]$.

The numerical values of $u(x)$ obtained with the MLS approximation and IIMLS method are shown in Figure 1 under regular and irregular node distributions, where the irregular node distribution is generated by adding a random

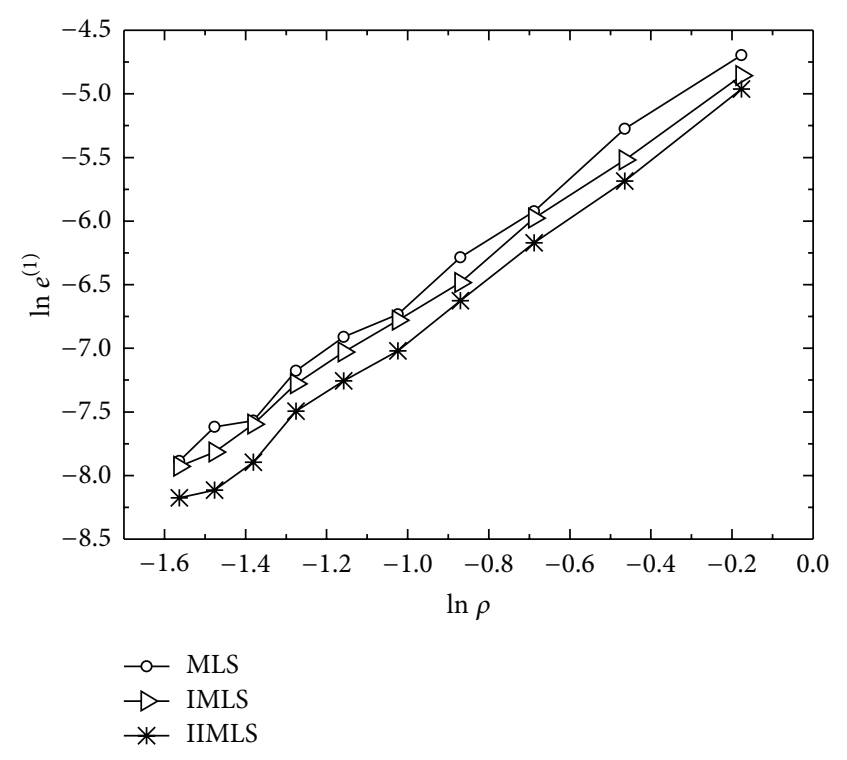

FIGURE 6: The error norms $e^{(1)}$ of the first derivative of $u(x)$ obtained with the MLS, IMLS, and IIMLS methods under the irregular node distribution.

perturbation on the regular node distribution. And the corresponding first derivatives are shown in Figure 2. It can be seen that the numerical results of the IIMLS method are in good agreement with the exact ones.

The error norms $e^{(0)}$ of the MLS, IMLS, and IIMLS methods under the regular and irregular node distributions are shown in Figures 3 and 4, respectively. It is shown that the shape functions of the IMLS and IIMLS methods all satisfy the property of Kronecker $\delta$ function.

The error norms $e^{(1)}$ of the MLS, IMLS, and IIMLS methods under the regular and irregular node distributions are shown in Figures 5 and 6, respectively. It is shown that the rates of convergence of the MLS, IMLS, and IIMLS methods are almost the same, and the error of the IIMLS method is less than that of the IMLS method. Hence, the IIMLS method has higher precision.

The second example is considered in the two-dimensional space, and the given function is $u\left(x_{1}, x_{2}\right)=e^{x_{1}} \sin x_{2}$, $\left(x_{1}, x_{2}\right) \in \Omega=[0,3] \times[0,3]$.

Under $21 \times 21$ regular and irregular node distributions, the numerical results of $u$ and $u_{2}$ at $x_{2}=3$ with the MLS approximation and IIMLS method are shown in Figures 7 and 8 , respectively. The irregular node distribution is generated by adding a random perturbation on the regular node distribution. It can also be seen that the numerical results of the IIMLS method are all in good agreement with the exact ones.

Under $11 \times 11,16 \times 16,21 \times 21$, and $26 \times 26$ irregular and regular node distributions, the error norms $e^{(0,0)}$ of the MLS, IMLS, and IIMLS methods are shown in Figures 9 and 10 , respectively. It is again evident that the shape functions of the IMLS and IIMLS methods all satisfy the property of Kronecker $\delta$ function. 


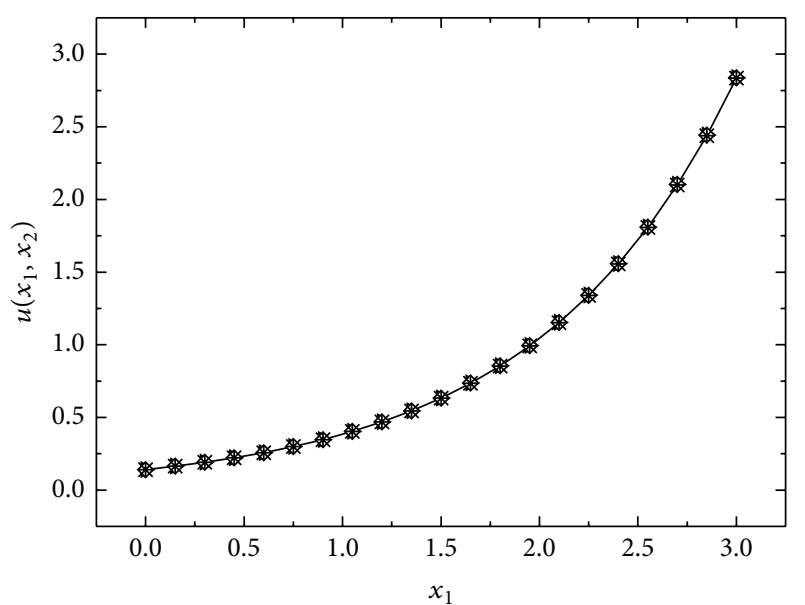

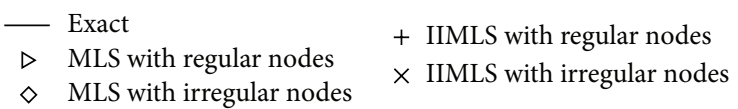

FIgURE 7: $u\left(x_{1}, x_{2}\right)$ at $x_{2}=3$ obtained with the MLS and IIMLS methods under regular and irregular node distributions.

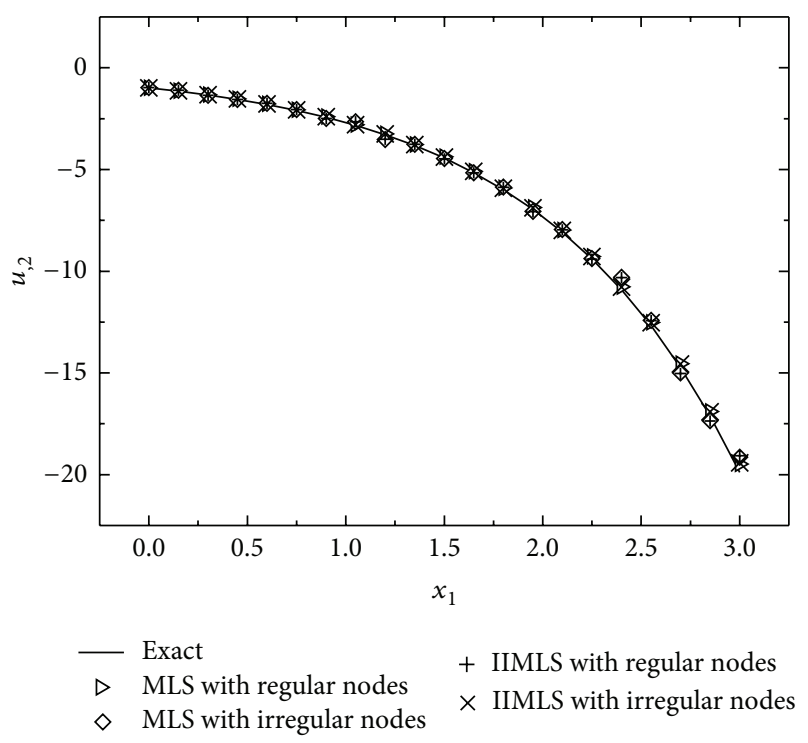

FIgURE 8: $u_{, 2}$ at $x_{2}=3$ obtained with the MLS and IIMLS methods under regular and irregular node distributions.

Under $11 \times 11,16 \times 16,21 \times 21$, and $26 \times 26$ regular node distributions, the error norms $e^{(1,0)}$ of the MLS, IMLS, and IIMLS methods are shown in Figure 11. Then by adding a random perturbation on the regular node distribution, the corresponding error norms $e^{(1,0)}$ under irregular node distribution are shown in Figure 12. It is shown that the rates of convergence of the MLS, IMLS, and IIMLS methods are almost the same. However, the error of the IIMLS method is less than that of the IMLS method and MLS approximation. Again, the IIMLS method has high precision.

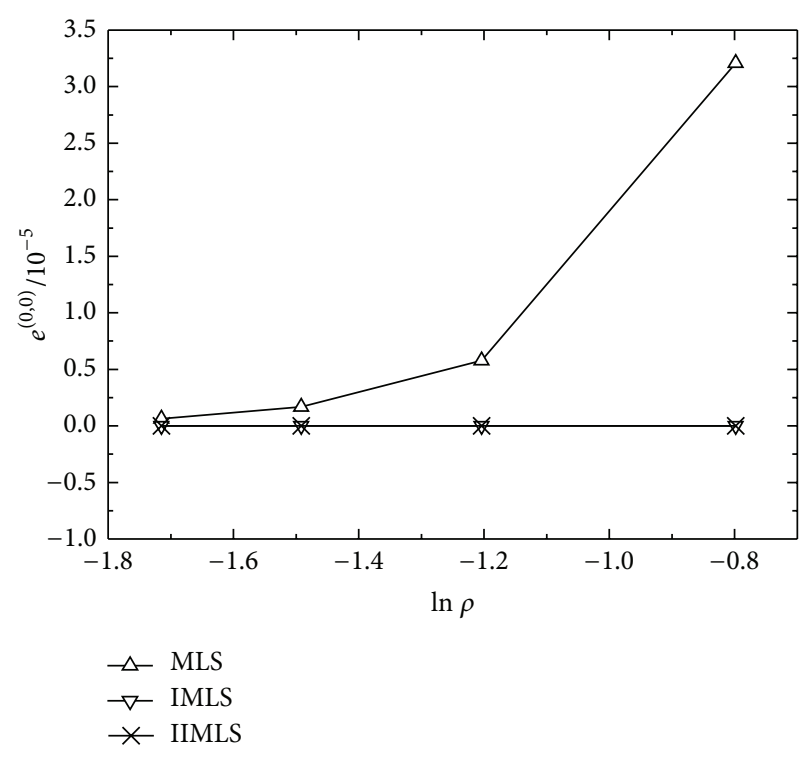

FIGURE 9: The error norms $e^{(0)}$ of $u\left(x_{1}, x_{2}\right)$ obtained with the MLS, IMLS, and IIMLS methods under the regular node distribution.

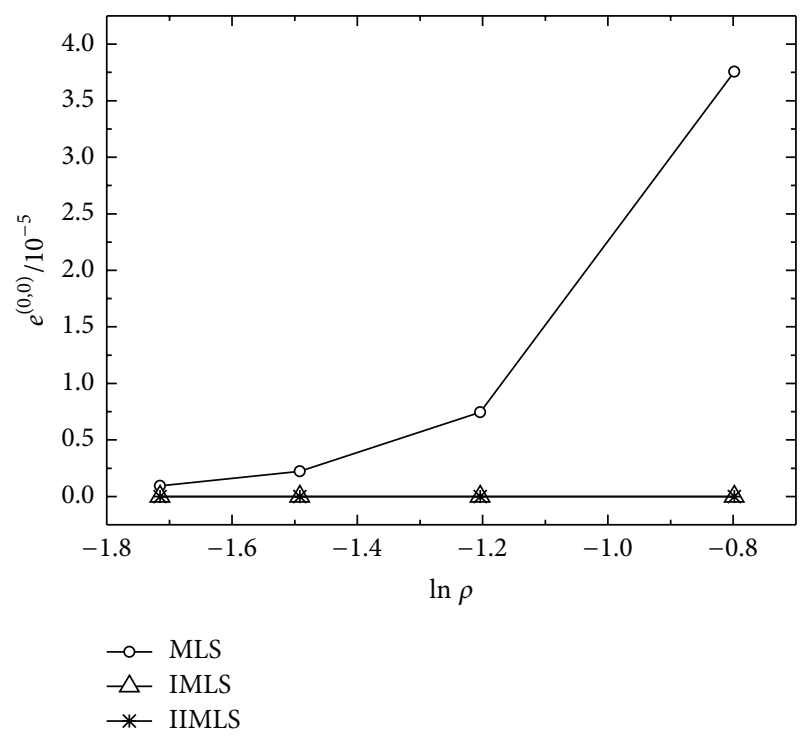

FIGURE 10: The error norms $e^{(0)}$ of $u\left(x_{1}, x_{2}\right)$ obtained with the MLS, IMLS, and IIMLS methods under the irregular node distribution.

4.2. Examples of the IIEFG Method. Two examples are selected to demonstrate the advantages of the IIEFG method for two-dimensional potential problems. The results obtained with the IIEFG method of this paper for these examples are compared with that obtained with the EFG and IEFG methods and analytical solutions. Define the error norm

$$
e_{T}=\frac{1}{K} \sqrt{\frac{\sum_{I=1}^{K}\left[\left[T_{I}^{h}-T_{I}\right]\right]^{2}}{\sum_{I=1}^{K}\left[T_{I}\right]^{2}}},
$$

where $K$ is the number of nodes and $T_{I}^{h}$ and $T_{I}$ are, respectively, the numerical and analytical solutions at nodes. 


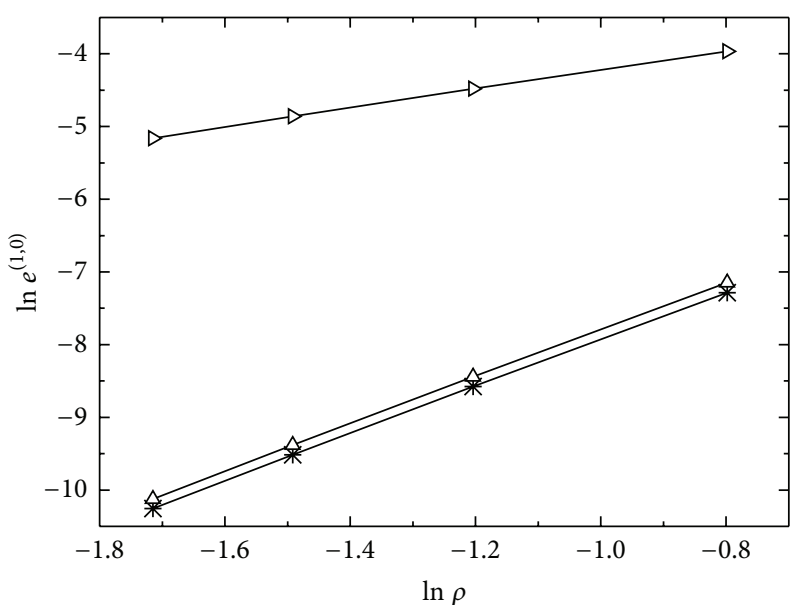

* IIMLS

$\triangle-$ MLS

$\rightarrow$ IMLS

FIGURE 11: The error norms $e^{(1,0)}$ of $u_{, 1}$ obtained with the MLS, IMLS, and IIMLS methods under regular node distribution.

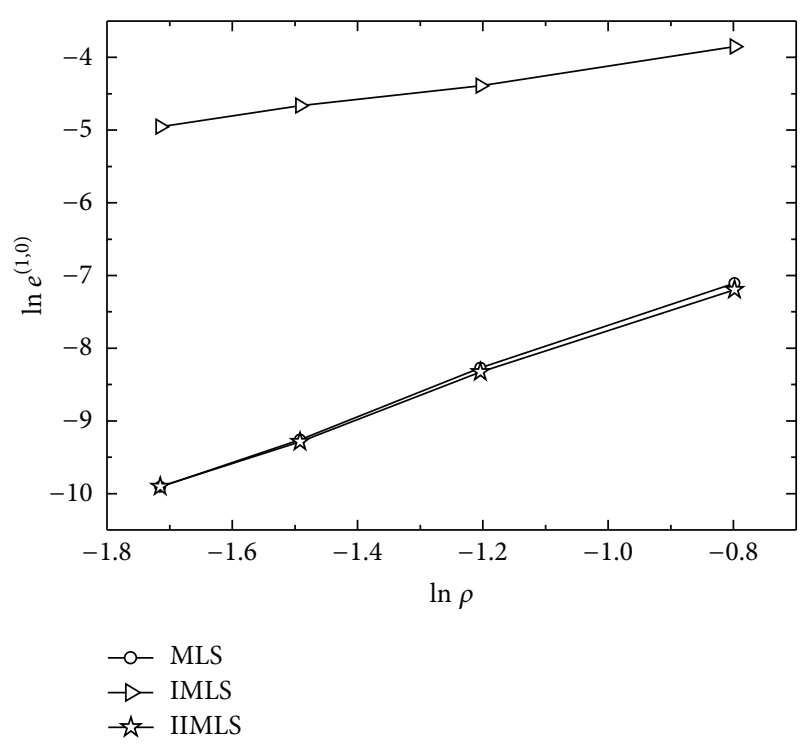

FIGURE 12: The error norms $e^{(1,0)}$ of $u_{, 1}$ obtained with the MLS, IMLS, and IIMLS methods under the irregular node distribution.

The third example considered is a temperature field of a rectangular plate governed by Laplace's equation

$$
\nabla^{2} T=\frac{\partial^{2} T}{\partial x_{1}^{2}}+\frac{\partial^{2} T}{\partial x_{2}^{2}}=0, \quad 0<x_{1}<5,0<x_{2}<10
$$

The boundary conditions are

$$
\begin{aligned}
& T\left(x_{1}, 0\right)=0, \quad 0<x_{1}<5, \\
& T\left(0, x_{2}\right)=0, \quad 0<x_{2}<10,
\end{aligned}
$$

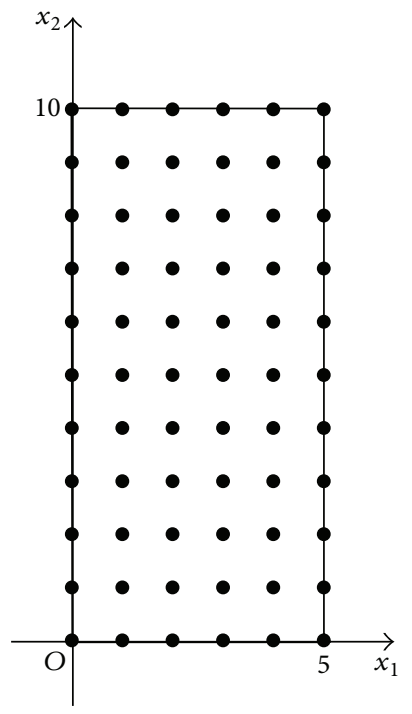

(a) Regular

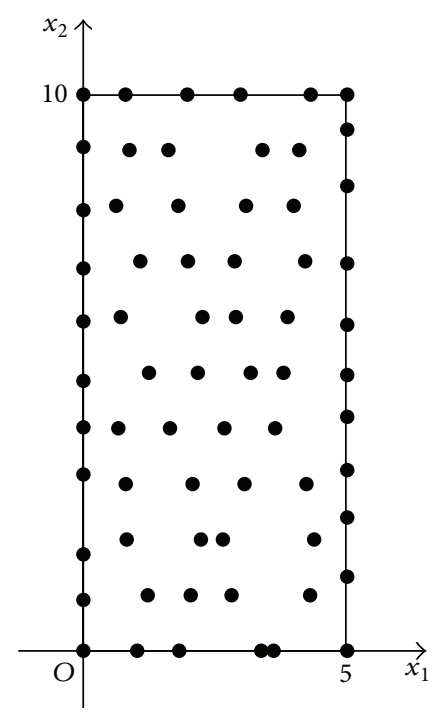

(b) Irregular
FIGURE 13: Node distribution.

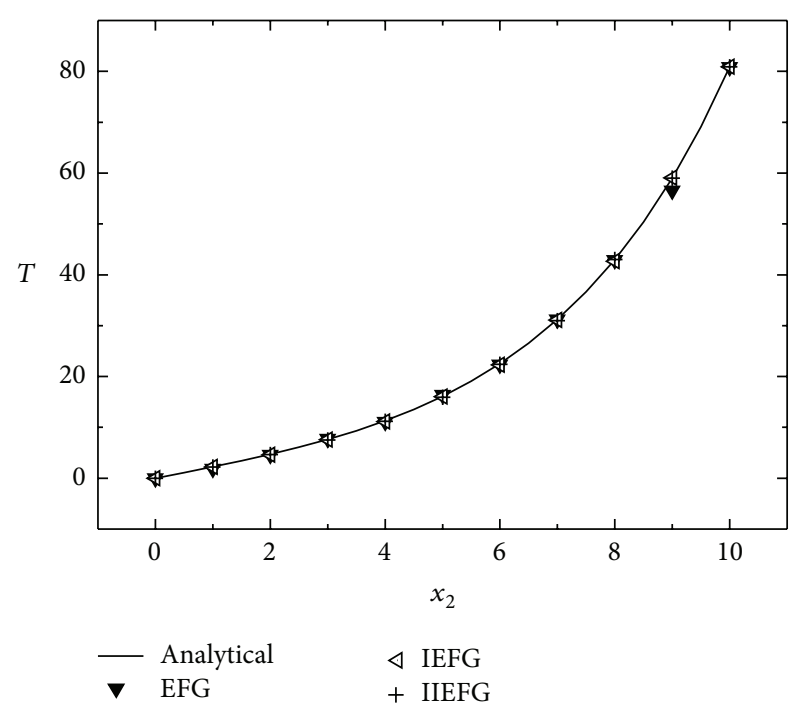

FIGURE 14: $T$ at $x_{1}=3$ obtained with the EFG, IEFG, and IIEFG methods under the regular node distribution.

$$
\begin{gathered}
T\left(x_{1}, 10\right)=100 \sin \left(\frac{\pi x_{1}}{10}\right), \quad 0<x_{1}<5, \\
\frac{\partial T\left(5, x_{2}\right)}{\partial x_{1}}=0, \quad 0<x_{2}<10 .
\end{gathered}
$$

The analytical solution for the temperature of this problem is

$$
u\left(x_{1}, x_{2}\right)=\frac{100 \sin \left(\pi x_{1} / 10\right) \sinh \left(\pi x_{2} / 10\right)}{\sinh (\pi)} .
$$

The linear basis function is used for analysis. When $6 \times 11$ regular node distribution is employed as shown 


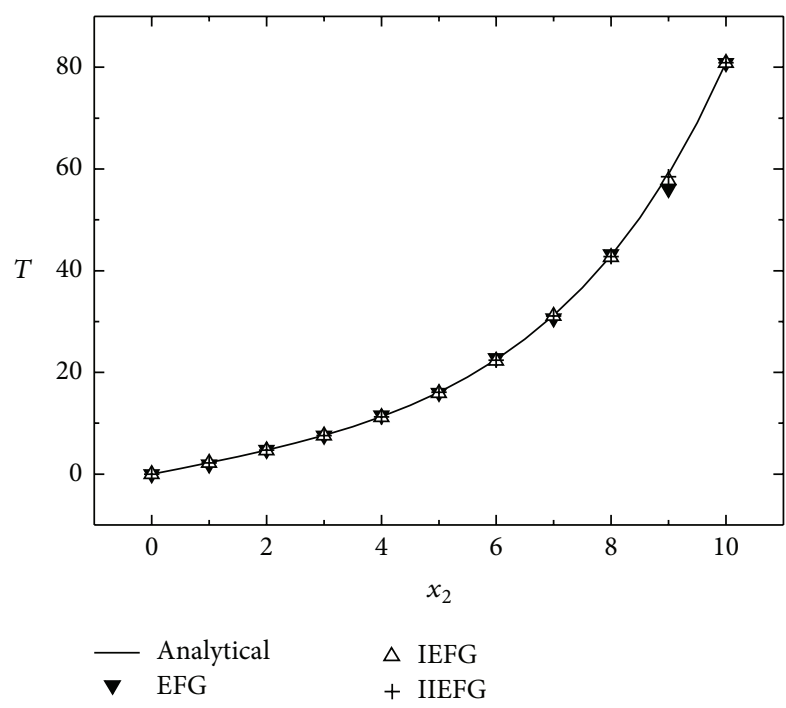

FIgure 15: $T$ at $x_{1}=3$ obtained with the EFG, IEFG, and IIEFG methods under the irregular node distribution.

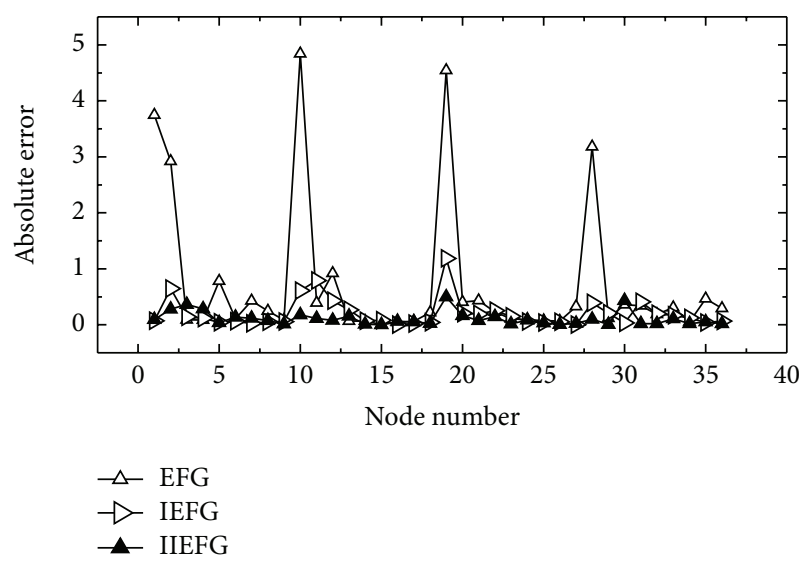

FIGURE 16: Absolute errors of $T$ at the inner nodes obtained with the EFG, IEFG, and IIEFG methods under the irregular node distribution.

in Figure 13(a), the temperatures $T$ at $x_{1}=3$ obtained with the IIEFG, EFG, and IEFG methods are shown in Figure 14. Then employing the irregular node distribution as shown in Figure 13(b), which is generated by adding a random perturbation on the regular node distribution, the temperatures $T$ at $x_{1}=3$ are shown in Figure 15, and the absolute errors at the inner nodes are shown in Figure 16. It is evident that the IIEFG method in this paper has higher computational precision than the EFG and IEFG methods.

Employing $5 \times 9,7 \times 13,9 \times 17$, and $11 \times 21$ regular node distributions, the error norms $e_{T}$ of temperature obtained with the IIEFG, EFG, and IEFG methods are shown in Figure 17. The average CPU times needed to furnish these results by using the IIEFG, EFG, and IEFG methods are respectively $10.95 \mathrm{~s}, 11.39 \mathrm{~s}$, and $11.04 \mathrm{~s}$. Then by adding a random perturbation on the regular node distribution, the error norms $e_{T}$ under the irregular node distribution are

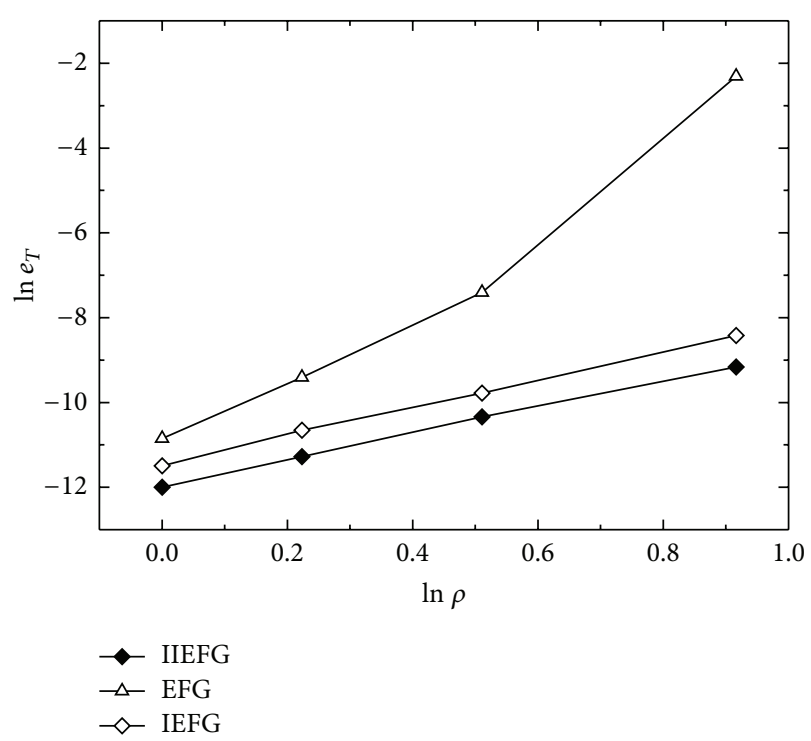

FIGURE 17: Error norms $e_{T}$ of $T$ obtained with the EFG, IEFG, and IIEFG methods under the regular node distribution.

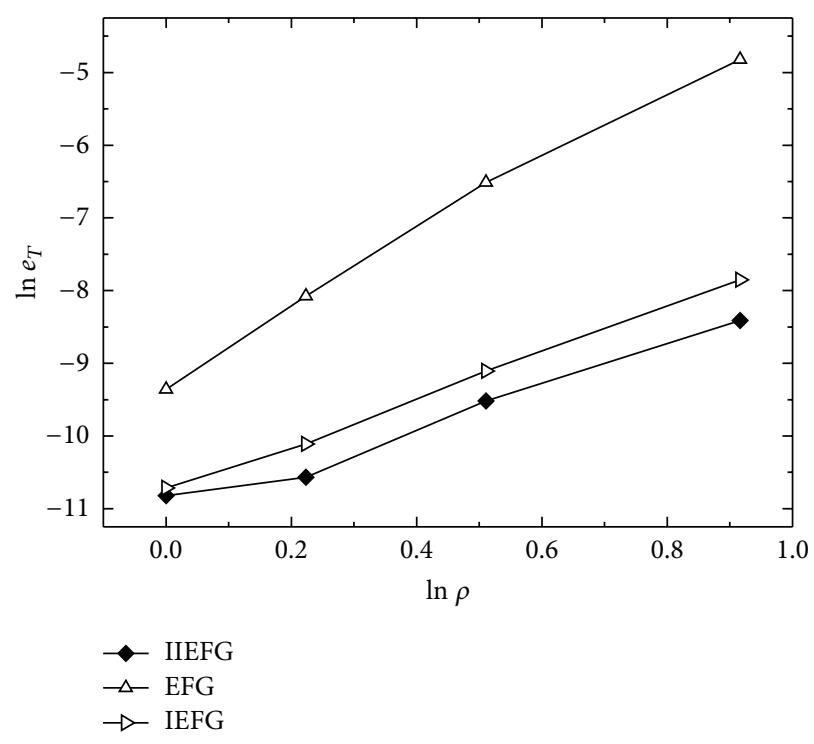

FIGURE 18: Error norms $e_{T}$ of $T$ obtained with the EFG, IEFG, and IIEFG methods under the irregular node distribution.

shown in Figure 18, and the average CPU times spent with the IIEFG, EFG, and IEFG methods are, respectively, $10.87 \mathrm{~s}$, $11.47 \mathrm{~s}$, and $11.09 \mathrm{~s}$. It can be seen that the IIEFG method has higher computational precision.

The fourth example considered is a temperature field of an annulus plate with inner radius $r_{1}$ and outer radius $r_{2}$ governed by Laplace's equation [58]:

$$
\nabla^{2} u=\frac{\partial^{2} u}{\partial x_{1}^{2}}+\frac{\partial^{2} u}{\partial x_{2}^{2}}=0
$$




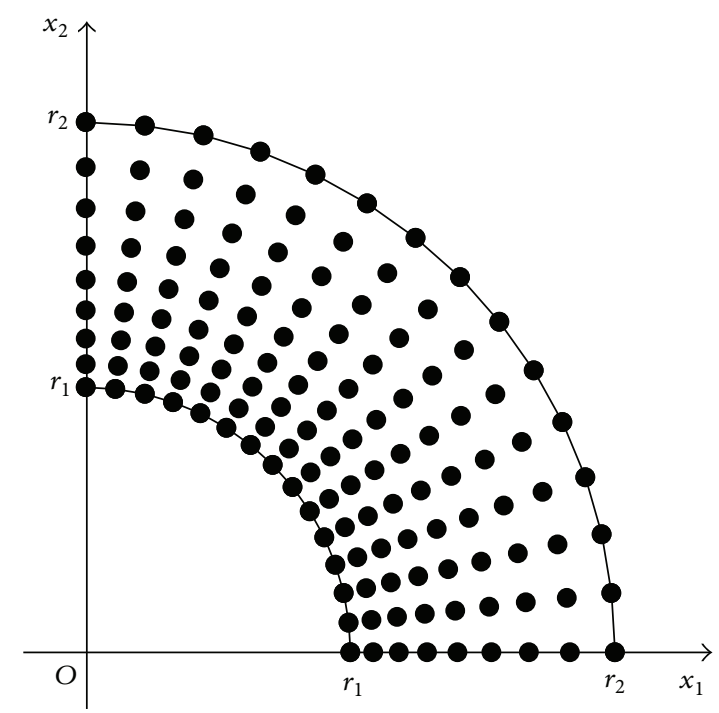

(a) Regular

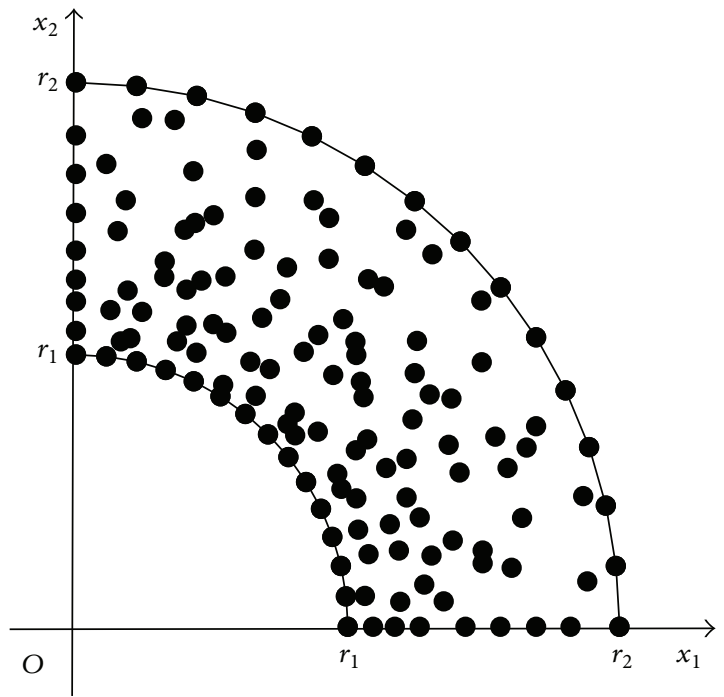

(b) Irregular

FIGURE 19: Node distribution.

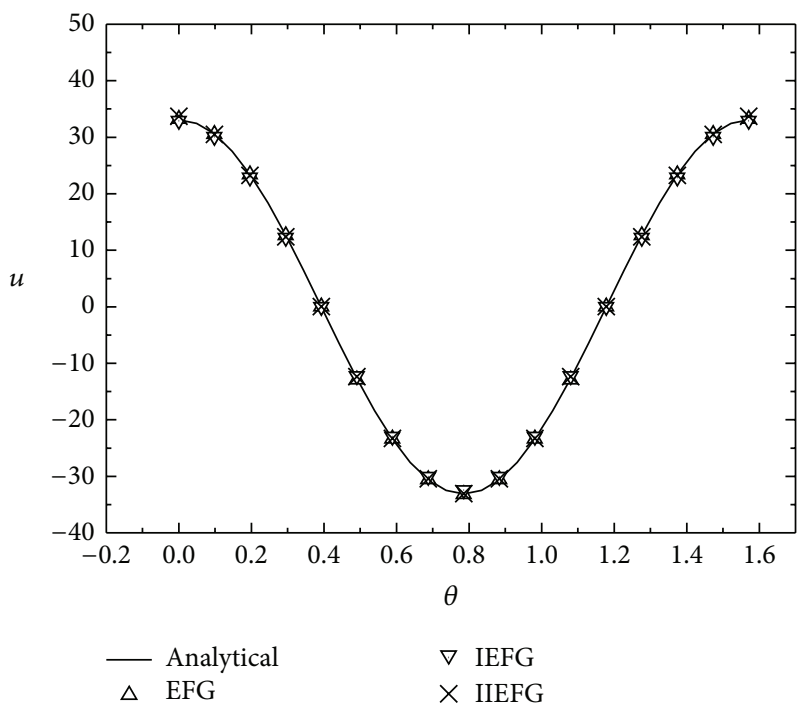

Figure 20: $u$ at $r=7.5$ obtained with the EFG, IEFG, and IIEFG methods under the regular node distribution.

Due to the symmetry of the model, only a quarter of the problem domain is modeled as shown in Figure 19. In the polar coordinate system $(r, \theta)$, the boundary conditions are

$$
\begin{aligned}
& u(r, \theta)=T_{1} \cos (4 \theta), \quad \text { on } r=r_{1}, \\
& u(r, \theta)=T_{2} \cos (4 \theta), \quad \text { on } r=r_{2} \\
& \frac{\partial u(r, \theta)}{\partial \theta}=0, \quad \text { on } \theta=0, \theta=\frac{\pi}{2}
\end{aligned}
$$

where $T_{1}$ and $T_{2}$ are given parameters.
The analytical solution is

$$
\begin{aligned}
& u(r, \theta) \\
& \quad=\frac{r_{1}^{4} r_{2}^{4}}{r_{2}^{8}-r_{1}^{8}}\left[T_{2}\left(\frac{r^{4}}{r_{1}^{4}}-\frac{r_{1}^{4}}{r^{4}}\right)-T_{1}\left(\frac{r^{4}}{r_{2}^{4}}-\frac{r_{2}^{4}}{r^{4}}\right)\right] \cos (4 \theta) .
\end{aligned}
$$

The parameters are taken as $r_{1}=5, r_{2}=10, T_{1}=50$, and $T_{2}=100$ in the computation. The regular and irregular node distributions that are used for the solution of this example are shown in Figure 19. Then the numerical solutions under the regular node distribution at $r=7.5$ are shown in Figure 20, and the average CPU times for obtaining the solutions with the IIEFG, EFG, and IEFG methods are, respectively, $0.81 \mathrm{~s}$, $1.16 \mathrm{~s}$, and $0.80 \mathrm{~s}$. Under the irregular node distribution, the temperatures along the arc $r=7.5$ and $r=9.5$ are shown in Figures 21 and 22, respectively. The average CPU time with the irregular node distribution is almost equal to that with the regular node, and their average CPU times of the IIEFG, EFG, and IEFG methods are, respectively, $0.81 \mathrm{~s}, 1.17 \mathrm{~s}$, and $0.80 \mathrm{~s}$. It can be seen that the IIEFG method has higher efficiency than the EFG method. And from these figures, it can also be observed that the IIEFG method in this paper has higher computational precision than the EFG and IEFG methods.

\section{Conclusions}

In this paper, based on the MLS approximation, the IIMLS method is presented. The shape function of the IIMLS method satisfies the property of Kronecker $\delta$ function. Then the meshless method based on the IIMLS method can apply the essential boundary condition directly and easily. Moreover, the number of unknown coefficients in the trial function of the IIMLS method is less than that in the trial function of the MLS approximation. Then fewer nodes are needed in 


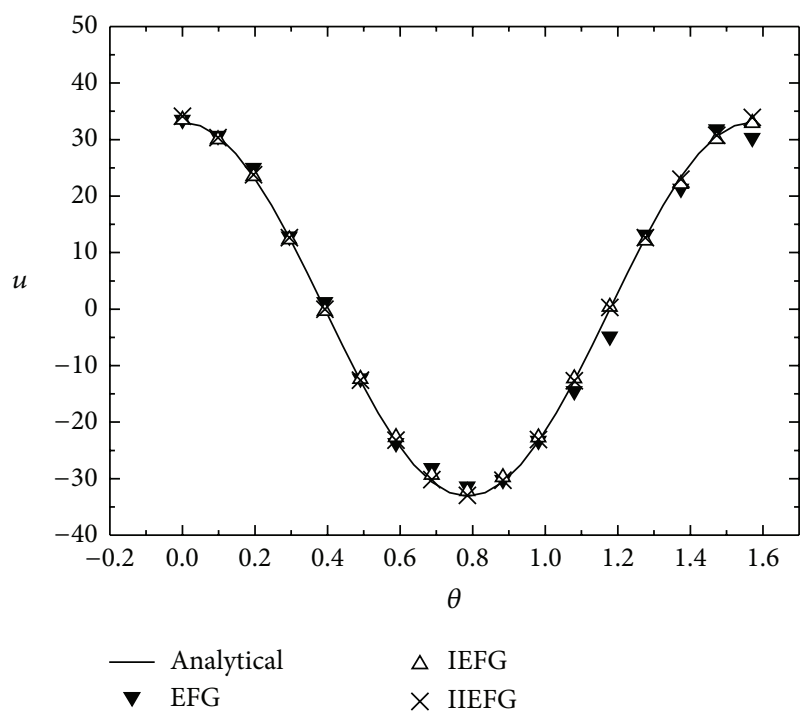

Figure 21: $u$ at $r=7.5$ obtained with the EFG, IEFG, and IIEFG methods under the irregular node distribution.

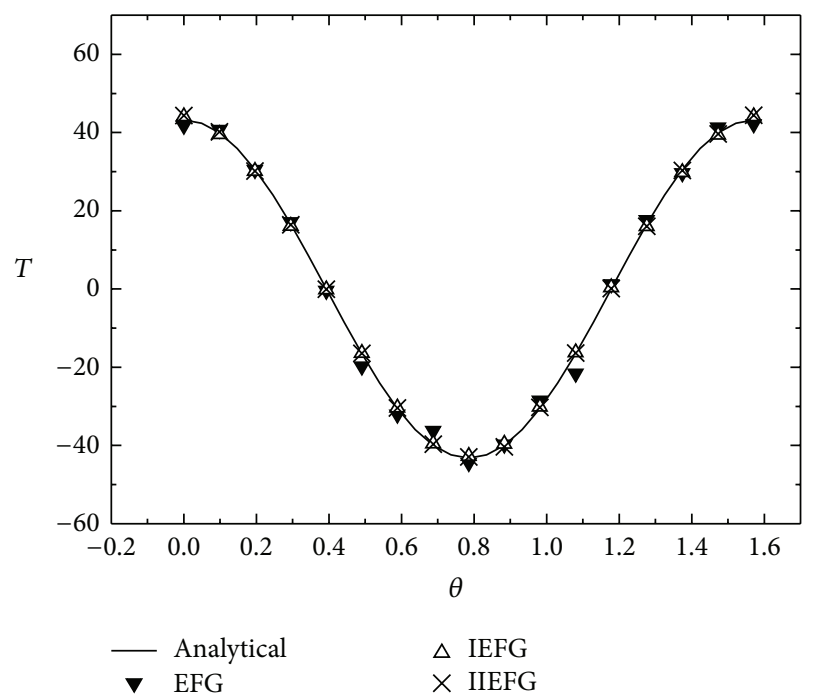

FIGURE 22: $u$ at $r=9.5$ obtained with the EFG, IEFG, and IIEFG methods under the irregular node distribution.

the local influence domain in the IIMLS method. Therefore, the IIMLS method has high computational precision. Compared with the IMLS method presented by Lancaster and Salkauskas, the weight function in the IIMLS method is nonsingular, and any weight function in the MLS approximation can be chosen as the weight function of the NMLS method. Then IIMLS method can overcome the difficulty caused by singularity of the weight function as in the IMLS method.

Based on the IIMLS and EFG methods, an IIEFG method for two-dimensional potential problems is presented. In the IIEFG method, the weight function is not singular, and the essential boundary conditions are applied naturally and directly. And there are fewer coefficients in the IIMLS method than in the MLS approximation; fewer nodes are needed in the entire domain in the IIEFG method formed from the IIMLS method than in the conventional EFG method. Then under the same node distribution, the IIEFG method in this paper has higher computational precision than the EFG and IEFG methods.

\section{Conflict of Interests}

The authors declare that there is no conflict of interests regarding the publication of this paper.

\section{Acknowledgments}

This work was supported by the National Natural Science Foundation of China (no. 11171208), Shanghai Leading Academic Discipline Project (no. S30106), and the Natural Science Foundation of Ningbo (no. 2013A610103).

\section{References}

[1] Z. Zhang, K. M. Liew, Y. Cheng, and Y. Y. Lee, "Analyzing 2D fracture problems with the improved element-free Galerkin method," Engineering Analysis with Boundary Elements, vol. 32, no. 3, pp. 241-250, 2008.

[2] K. M. Liew, J. Ren, and J. N. Reddy, "Numerical simulation of thermomechanical behaviours of shape memory alloys via a non-linear mesh-free Galerkin formulation," International Journal for Numerical Methods in Engineering, vol. 63, no. 7, pp. 1014-1040, 2005.

[3] D. Li, F. Bai, Y. Cheng, and K. M. Liew, "A novel complex variable element-free Galerkin method for two-dimensional large deformation problems," Computer Methods in Applied Mechanics and Engineering, vol. 233-236, pp. 1-10, 2012.

[4] T. Belytschko, Y. Y. Lu, and L. Gu, "Element-free Galerkin methods," International Journal for Numerical Methods in Engineering, vol. 37, no. 2, pp. 229-256, 1994.

[5] W. Ju-Feng, S. Feng-Xin, and C. Rong-Jun, "Element-free Galerkin method for a kind of KdV equation," Chinese Physics $B$, vol. 19, no. 6, Article ID 060201, 2010.

[6] Z. Zhang, D.-M. Li, Y.-M. Cheng, and K. M. Liew, "The improved element-free Galerkin method for three-dimensional wave equation," Acta Mechanica Sinica, vol. 28, no. 3, pp. 808818, 2012.

[7] Z. Zhang, J. F. Wang, Y. M. Cheng, and K. M. Liew, "The improved element-free Galerkin method for three-dimensional transient heat conduction problems," Science China Physics, Mechanics \& Astronomy, vol. 56, no. 8, pp. 1568-1580, 2013.

[8] Z. Zhang, S. Y. Hao, K. M. Liew, and Y. M. Cheng, "The improved element-free Galerkin method for two-dimensional elastodynamics problems," Engineering Analysis with Boundary Elements, vol. 37, no. 12, pp. 1576-1584, 2013.

[9] S. N. Atluri and T. Zhu, "A new meshless local Petrov-Galerkin (MLPG) approach in computational mechanics," Computational Mechanics, vol. 22, no. 2, pp. 117-127, 1998.

[10] L. Chen and Y. M. Cheng, "Reproducing kernel particle method with complex variables for elasticity," Acta Physica Sinica, vol. 57, no. 1, pp. 1-10, 2008.

[11] L. Chen and Y. M. Cheng, "Complex variable reproducing kernel particle method for transient heat conduction problems," Acta Physica Sinica, vol. 57, no. 10, pp. 6047-6055, 2008. 
[12] L. Chen and Y. M. Cheng, "The complex variable reproducing kernel particle method for elasto-plasticity problems," Science China, vol. 53, no. 5, pp. 954-965, 2010.

[13] L. Chen and Y.-M. Cheng, "The complex variable reproducing kernel particle method for two-dimensional elastodynamics," Chinese Physics B, vol. 19, no. 9, Article ID 090204, 2010.

[14] Y. M. Cheng, M. J. Peng, and J. H. Li, "The complex variable moving least-square approximation and its application," Chinese Journal of Theoretical and Applied Mechanics, vol. 37, no. 6, pp. 719-723, 2005.

[15] Y. M. Cheng and J. H. Li, "A meshless method with complex variables for elasticity," Acta Physica Sinica, vol. 54, no. 10, pp. 4463-4471, 2005.

[16] Y. Cheng and J. Li, "Complex variable meshless method for fracture problems," Science in China, Series G, vol. 49, no. 1, pp. 46-59, 2006.

[17] K. M. Liew, C. Feng, Y. Cheng, and S. Kitipornchai, "Complex variable moving least-squares method: a meshless approximation technique," International Journal for Numerical Methods in Engineering, vol. 70, no. 1, pp. 46-70, 2007.

[18] M. Peng, P. Liu, and Y. Cheng, "The complex variable elementfree Galerkin (CVEFG) method for two-dimensional elasticity problems," International Journal of Applied Mechanics, vol. 1, no. 2, pp. 367-385, 2009.

[19] M. Peng, D. Li, and Y. Cheng, "The complex variable elementfree Galerkin (CVEFG) method for elasto-plasticity problems," Engineering Structures, vol. 33, no. 1, pp. 127-135, 2011.

[20] F.-N. Bai, D.-M. Li, J.-F. Wang, and Y.-M. Cheng, "An improved complex variable element-free Galerkin method for twodimensional elasticity problems," Chinese Physics B, vol. 21, no. 2, Article ID 020204, 2012.

[21] F.-N. Bai, D.-M. Li, J.-F. Wang, and Y.-M. Cheng, "An improved complex variable element-free Galerkin method for twodimensional elasticity problems," Chinese Physics B, vol. 21, no. 2, Article ID 020204, 2012.

[22] Y. M. Cheng, R. X. Li, and M. J. Peng, "Complex variable element-free Galerkin (CVEFG) method for viscoelasticity problems," Chinese Physics B, vol. 21, no. 9, Article ID 090205, 2012.

[23] Y. M. Cheng, J. F. Wang, and R. X. Li, “The complex variable element-free Galerkin (CVEFG) method for two-dimensional elastodynamics problems," International Journal of Applied Mechanics, vol. 4, no. 4, Article ID 1250042, 2012.

[24] S. C. Li and Y. M. Cheng, "Meshless numerical manifold method based on unit partition," Acta Mechanica Sinica, vol. 36, no. 4, pp. 496-500, 2004.

[25] S. C. Li and Y. M. Cheng, "Numerical manifold method and its applications in rock mechanics," Advances in Mechanics, vol. 34, no. 4, pp. 446-454, 2004.

[26] S. Li, Y. Cheng, and Y.-F. Wu, "Numerical manifold method based on the method of weighted residuals," Computational Mechanics, vol. 35, no. 6, pp. 470-480, 2005.

[27] S. C. Li, S. C. Li, and Y. M. Cheng, "Enriched meshless manifold method for two-dimensional crack modeling," Theoretical and Applied Fracture Mechanics, vol. 44, no. 3, pp. 234-248, 2005.

[28] S.-C. Li, Y.-M. Cheng, and S.-C. Li, "Meshless manifold method for dynamic fracture mechanics," Acta Physica Sinica, vol. 55, no. 9, pp. 4760-4766, 2006.

[29] H. F. Gao and Y. M. Cheng, "Complex variable numerical manifold method for elasticity," Chinese Journal of Theoretical and Applied Mechanics. Lixue Xuebao, vol. 41, no. 4, pp. 480488, 2009.
[30] H. Gao and Y. Cheng, "A complex variable meshless manifold method for fracture problems," International Journal of Computational Methods, vol. 7, no. 1, pp. 55-81, 2010.

[31] R. J. Cheng and K. M. Liew, "Analyzing two-dimensional sine-Gordon equation with the mesh-free reproducing kernel particle Ritz method," Computer Methods in Applied Mechanics and Engineering, vol. 245-246, pp. 132-143, 2012.

[32] E. Oñate, S. Idelsohn, O. C. Zienkiewicz, and R. L. Taylor, "A finite point method in computational mechanics. Applications to convective transport and fluid flow," International Journal for Numerical Methods in Engineering, vol. 39, no. 22, pp. 38393866, 1996.

[33] M. Dehghan and M. Tatari, "Determination of a control parameter in a one-dimensional parabolic equation using the method of radial basis functions," Mathematical and Computer Modelling, vol. 44, no. 11-12, pp. 1160-1168, 2006.

[34] B. D. Dai and Y. M. Cheng, "Local boundary integral equation method based on radial basis functions for potential problems," Acta Physica Sinica, vol. 56, no. 2, pp. 597-603, 2007.

[35] Y. M. Cheng and M. J. Chen, "A boundary element-free method for linear elasticity," Acta Mechanica Sinica, vol. 35, no. 2, pp. 181-186, 2003.

[36] Y. Cheng and M. Peng, "Boundary element-free method for elastodynamics," Science in China G, vol. 48, no. 6, pp. 641-657, 2005.

[37] K. M. Liew, Y. Cheng, and S. Kitipornchai, "Boundary elementfree method (BEFM) for two-dimensional elastodynamic analysis using Laplace transform," International Journal for Numerical Methods in Engineering, vol. 64, no. 12, pp. 1610-1627, 2005.

[38] K. M. Liew and Y. Cheng, "Complex variable boundary element-free method for two-dimensional elastodynamic problems," Computer Methods in Applied Mechanics and Engineering, vol. 198, no. 49-52, pp. 3925-3933, 2009.

[39] M. Peng and Y. Cheng, "A boundary element-free method (BEFM) for two-dimensional potential problems," Engineering Analysis with Boundary Elements, vol. 33, no. 1, pp. 77-82, 2009.

[40] Y. Cheng, K. M. Liew, and S. Kitipornchair, "Reply to 'Comments on Boundary element-free method (BEFM) and its application to two-dimensional elasticity problems," International Journal for Numerical Methods in Engineering, vol. 78, no. 10, pp. 1258-1260, 2009.

[41] J. Zhang, M. Tanaka, and T. Matsumoto, "Meshless analysis of potential problems in three dimensions with the hybrid boundary node method," International Journal for Numerical Methods in Engineering, vol. 59, no. 9, pp. 1147-1166, 2004.

[42] S. N. Atluri, J. Sladek, V. Sladek, and T. Zhu, "Local boundary integral equation (LBIE) and it's meshless implementation for linear elasticity," Computational Mechanics, vol. 25, no. 2, pp. 180-198, 2000.

[43] B. Dai and Y. Cheng, "An improved local boundary integral equation method for two-dimensional potential problems," International Journal of Applied Mechanics, vol. 2, no. 2, pp. 421436, 2010.

[44] D. Shepard, "A two-dimensional interpolation function for irregularly spaced points," in Proceeding of the $23 \mathrm{rd}$ ACM National Conference, pp. 517-524, 1968.

[45] P. Lancaster and K. Salkauskas, "Surfaces generated by moving least squares methods," Mathematics of Computation, vol. 37, no. 155, pp. 141-158, 1981. 
[46] T. Zhu and S. N. Atluri, "A modified collocation method and a penalty formulation for enforcing the essential boundary conditions in the element free Galerkin method," Computational Mechanics, vol. 21, no. 3, pp. 211-222, 1998.

[47] T. Mostt and C. Bucher, "A Moving Least Squares weighting function for the Element-free Galerkin Method which almost fulfills essential boundary conditions," Structural Engineering and Mechanics, vol. 21, no. 3, pp. 315-332, 2005.

[48] T. Most and C. Bucher, "New concepts for moving least squares: an interpolating non-singular weighting function and weighted nodal least squares," Engineering Analysis with Boundary Elements, vol. 32, no. 6, pp. 461-470, 2008.

[49] S. L. L. Verardi, J. M. Machado, and Y. Shiyou, "The application of interpolating MLS approximations to the analysis of MHD flows," Finite Elements in Analysis and Design, vol. 39, no. 12, pp. 1173-1187, 2003.

[50] I. Kaljević and S. Saigal, "An improved element free Galerkin formulation," International Journal for Numerical Methods in Engineering, vol. 40, no. 16, pp. 2953-2974, 1997.

[51] H.-P. Ren, Y.-M. Cheng, and W. Zhang, "An improved boundary element-free method (IBEFM) for two-dimensional potential problems," Chinese Physics B, vol. 18, no. 10, pp. 4065-4073, 2009.

[52] R. Hongping, C. Yumin, and Z. Wu, "An interpolating boundary element-free method (IBEFM) for elasticity problems," Science China, vol. 53, no. 4, pp. 758-766, 2010.

[53] H. Ren and Y. Cheng, "The interpolating element-free Galerkin (IEFG) method for two-dimensional potential problems," Engineering Analysis with Boundary Elements, vol. 36, no. 5, pp. 873880, 2012.

[54] H. Ren and Y. Cheng, "The interpolating element-free Galerkin (IEFG) method for two-dimensional potential problems," Engineering Analysis with Boundary Elements, vol. 36, no. 5, pp. 873880, 2012.

[55] H. Netuzhylov, "Enforcement of boundary conditions in meshfree methods using interpolating moving least squares," Engineering Analysis with Boundary Elements, vol. 32, no. 6, pp. 512516, 2008.

[56] J. F. Wang, F. X. Sun, and Y. M. Cheng, "An improved interpolating element-free Galerkin method with a nonsingular weight function for two-dimensional potential problems," Chinese Physics B, vol. 21, no. 9, Article ID 090204, 2012.

[57] J. Wang, J. Wang, F. Sun, and Y. Cheng, "An interpolating boundary element-free method with nonsingular weight function for two-dimensional potential problems," International Journal of Computational Methods, vol. 10, no. 6, Article ID 1350043, 2013.

[58] A. P. S. Selvadurai, Partial Differential Equations in Mechanics, Springer, Berlin, Germany, 2000. 


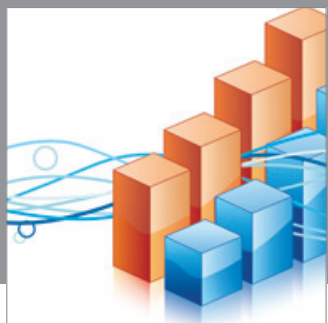

Advances in

Operations Research

mansans

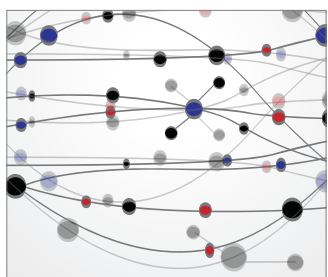

The Scientific World Journal
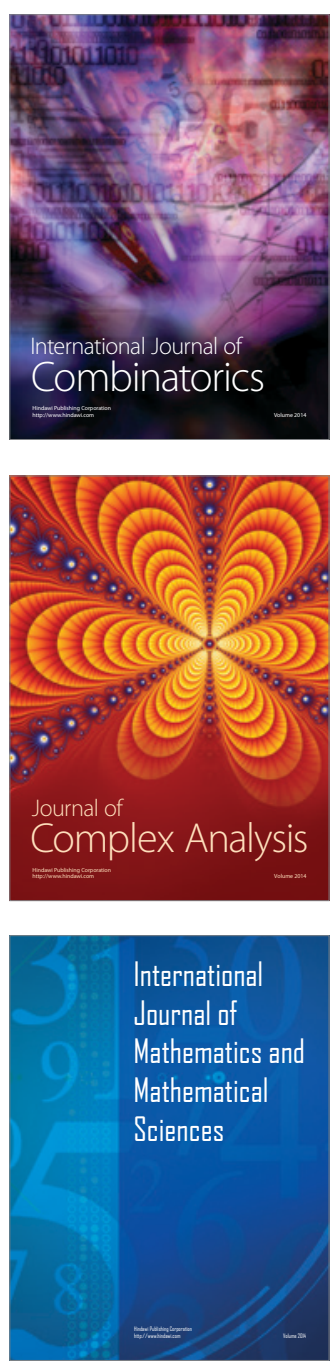
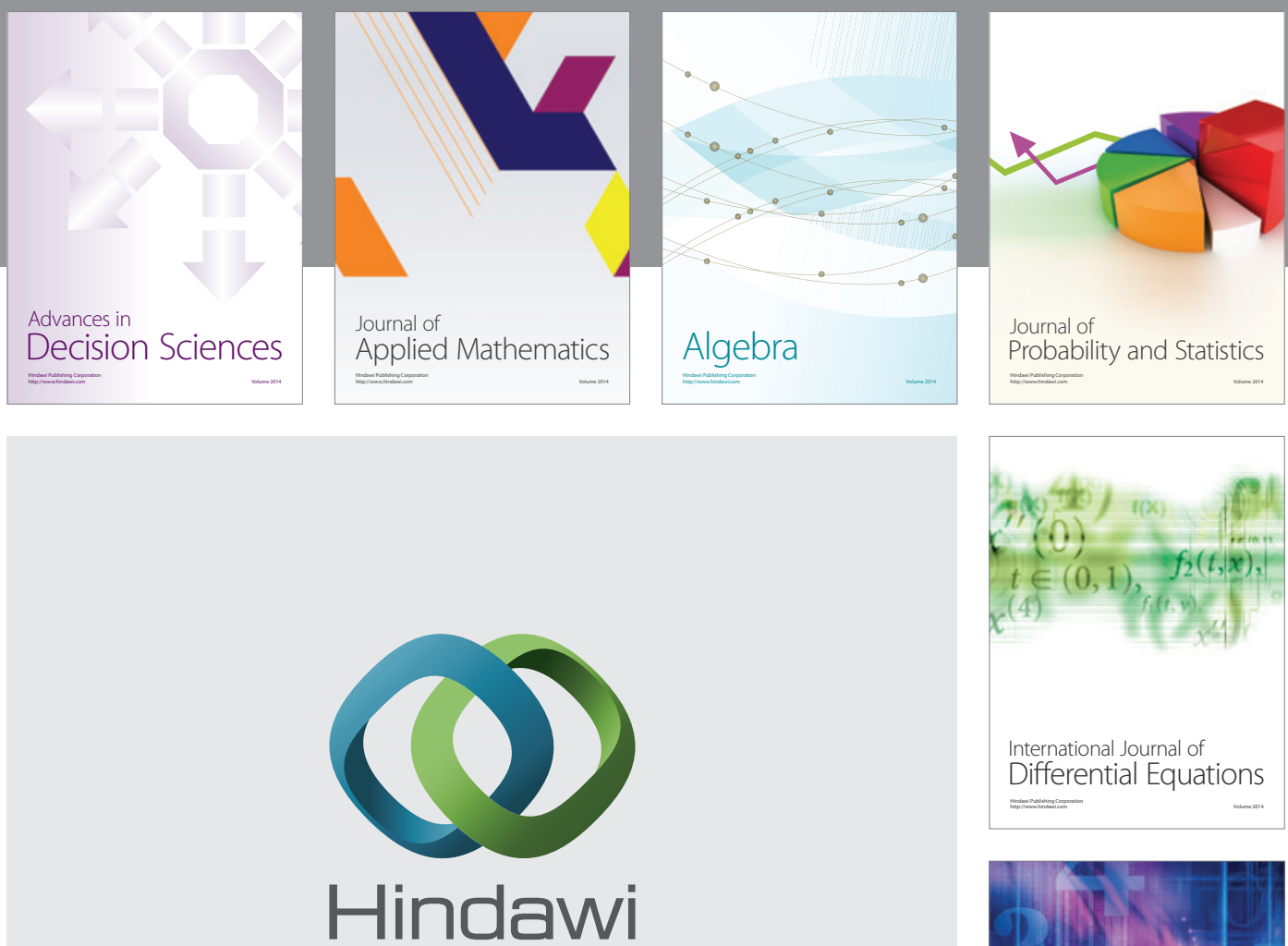

Submit your manuscripts at http://www.hindawi.com
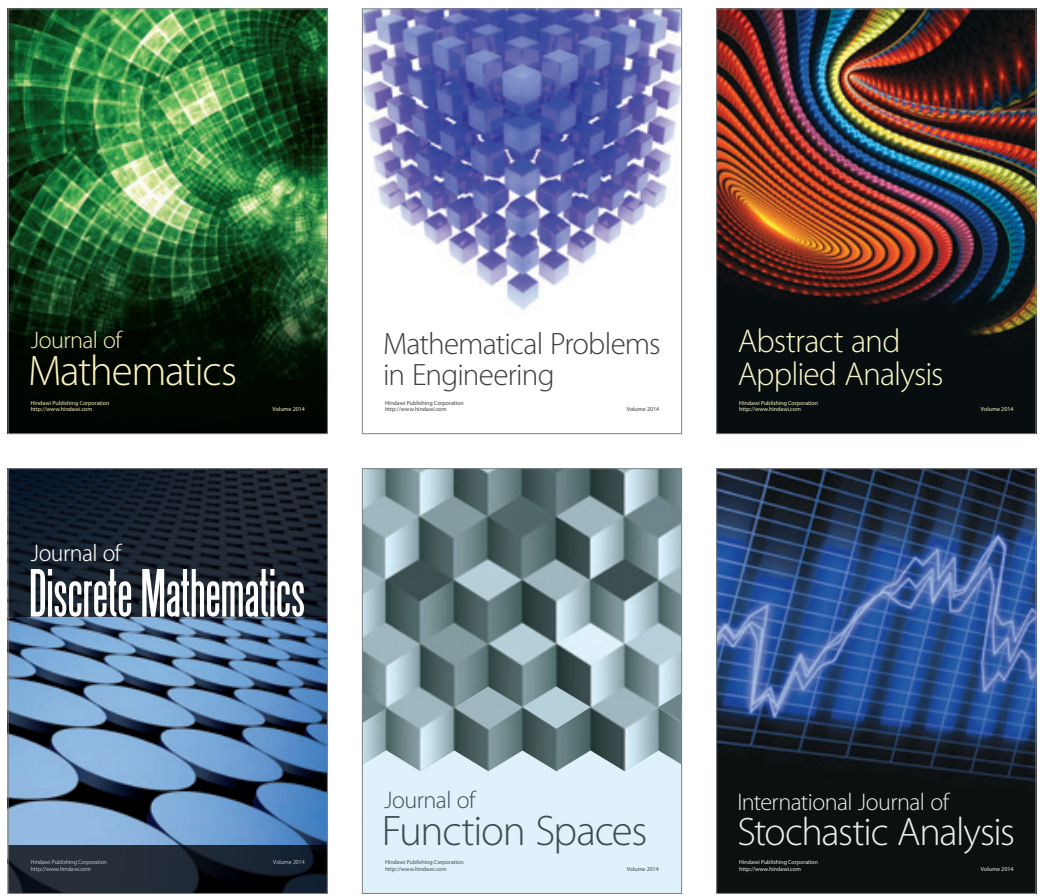

Journal of

Function Spaces

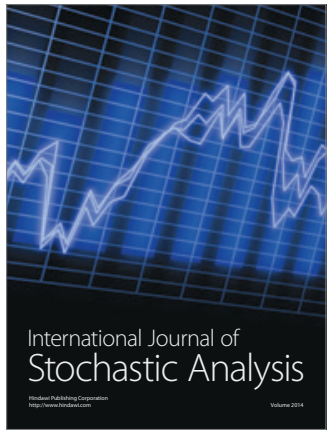

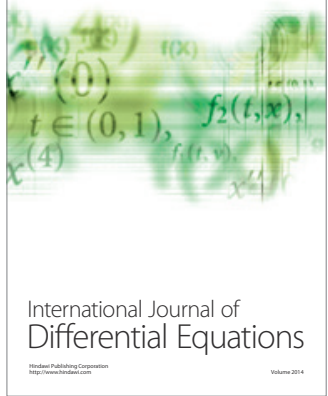
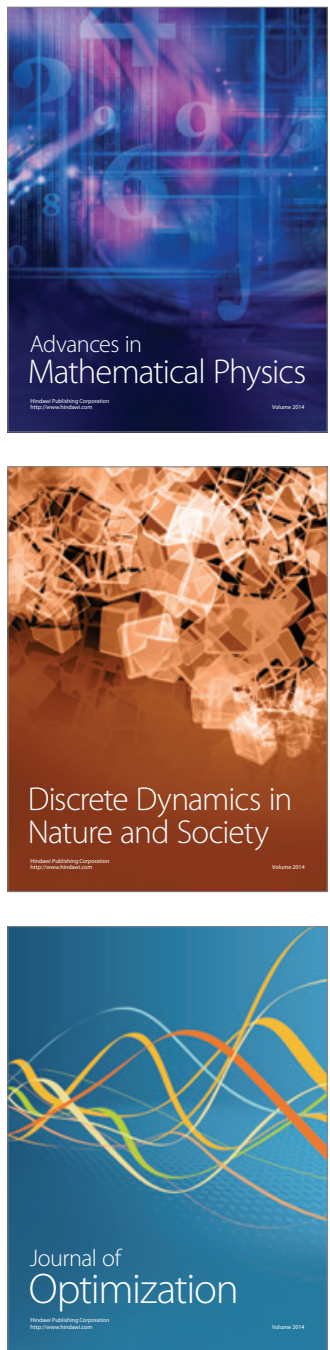